https://doi.org/10.15407/ujpe63.7.623

D. FISCALETTI, ${ }^{1}$ A. SORLI ${ }^{2}$

${ }^{1}$ SpaceLife Institute, San Lorenzo in Campo (PU), Italy

(Italy; e-mail: spacelife.institute@gmail.com)

${ }^{2}$ Foundations of Physics Institute, Idrija, Slovenia

(Slovenia; e-mail: sorli.amrit@gmail.com)

\title{
QUANTUM RELATIVITY: VARIABLE ENERGY DENSITY OF QUANTUM VACUUM AS THE ORIGIN OF MASS, GRAVITY, AND THE QUANTUM BEHAVIOR
}

\begin{abstract}
In Quantum Relativity, time and space are separated. Time is the numerical order of material changes, and space is the medium, in which these changes take place. Space has the origin in a three-dimensional quantum vacuum defined by fluctuations of the energy density corresponding to elementary $\boldsymbol{R} \boldsymbol{S}$ (reduction state) processes of creation/annihilation of elementary quanta. Quantum Relativity provides a unifying approach to special relativity, general relativity, and quantum mechanics. Each physical object from the micro- to the macroscale can be derived from an opportune diminishing of the quantum vacuum energy density. In particular, the variable energy density of space in Quantum Relativity corresponds to the curvature of space in general relativity. In quantum theory, the behavior of each subatomic particle follows from opportune elementary $\boldsymbol{R} \boldsymbol{S}$ processes of creation/annihilation of quanta guided by a quantum potential of the vacuum. Finally, the perspectives of this model regarding the view of gravity and quantum as two aspects of the same coin and the electroweak scale are analyzed.

Keywords: mass, gravity, General Relativity, Quantum Relativity, quantum vacuum, quantum mechanics, Standard Model.
\end{abstract}

\section{Introduction}

The $20^{\text {th }}$ century theoretical physics replaced the notion of an "empty" space with the idea of a unified quantum vacuum as a fundamental medium subtending the observable forms of matter, energy, and spacetime. As a consequence of quantum field theories and cosmology, the physical vacuum emerges as a unified system governing the processes taking place in the micro- and macroworlds, which manifests itself on all space-time scales. The real particles such as electrons, positrons, photons, hadrons, etc., as well as all macroscopic bodies, are quantum wave-like excitations of this medium.

The missing point inside the physics of the $20^{\text {th }}$ century is that a region of universal space, which is

(C) D. FISCALETTI, A. SORLI, 2018 void theoretically of all fields, elementary particles, and massive objects, still exists on its own and so must have some specific physical origin. The so-called "empty space" is a type of energy that is "full" of itself and has its independent physical existence. We explored recently this possibility by introducing, on the basis of the Planckian metric emerging, for example, from loop quantum gravity [1-3], a model of a three-dimensional (3D) dynamic quantum vacuum, which can create a bridge between quantum mechanics and general relativity. In this model, the background space of physical processes is a $3 \mathrm{D}$ quantum vacuum, where each elementary particle is determined by elementary reduction-state (RS) processes of creation/annihilation of quanta (more precisely, of virtual particle-antiparticle pairs) corresponding to opportune changes of the energy density of space [4, 
5]. This model, as it provides a natural improvement of general relativity and quantum theory with the help of relevant foundations of quantum field theory regarding quantum vacuum properties, can be appropriately named Quantum Relativity.

In this approach, the variable energy density of space is the fundamental energy, which gives origin to the different physical entities existing in the universe. In the outer intergalactic space, namely in the absence of material objects, the energy density of space is defined by the Planck energy density:

$\rho_{\mathrm{PE}}=\frac{m_{\mathrm{p}} c^{2}}{l_{\mathrm{p}}^{3}}$,

where $m_{\mathrm{P}}$ is Planck's mass, $c$ is the light speed, and $l_{\mathrm{p}}$ is Planck's length. Quantity (1) is the maximum value of the energy density of space and physically corresponds to the total average volumetric energy density, owed to all the frequency modes possible within the visible size of the universe, expressed by

$\rho_{\mathrm{PE}}=\frac{c^{7}}{\hbar G^{2}} \approx 4.641266 \times 10^{113} \mathrm{~J} / \mathrm{m}^{3}$,

$\hbar$ being Planck's reduced constant, $G$ the universal gravitation constant. In the outer intergalactic space, the curvature of space is zero, and its energy density corresponds to value (2). The Planck energy density is the average energy density of the empty universal space, in which there are no material objects. Out of this fundamental energy pool of the universe, particles and antiparticles continuously appear and disappear.

The quantum vacuum characterized by the energy density (1) identifies a 3D Euclid space as a preferred fundamental arena, which is quantitatively defined by Galilean transformations for the three spatial dimensions

$$
\begin{aligned}
& X^{\prime}=X-v \tau, \\
& Y^{\prime}=Y, \\
& Z^{\prime}=Z,
\end{aligned}
$$

and Selleri's transformation

$$
\tau^{\prime}=\sqrt{1-\frac{v^{2}}{c^{2}}} \tau
$$

for the rate of clocks. In Eqs. (3) and (4), $v$ is the velocity of the moving observer $O^{\prime}$ of the inertial frame $o^{\prime}$ measured by the stationary observer $O$, and $\tau$ is the proper time of the observer $O$ of the rest frame $o$, namely the speed of clocks of the observer $O$ (here, as usual, the origin of $o^{\prime}$, observed from $o$, is seen to move with velocity $v$ parallel to the $X$ axis). According to transformations (3) and (4), the temporal coordinate has thus a different ontological status with respect to the spatial coordinates. It is the motion relative to the rest frame of the Euclidean space associated with the quantum vacuum energy density (1) that influences clocks' running. The duration of material changes satisfying the standard Lorentz transformation for the temporal coordinate is a physical scaling function, which emerges from the more fundamental numerical order $\tau$ defined by the relation

$t=\tau\left(1-\frac{v^{2}}{c^{2}}\right)+\frac{v X}{c^{2}}$,

and determines itself a the re-scaling factor of the distance in the first spatial coordinate determined by the material motion of the form

$\delta=\sqrt{\frac{1}{\tau}\left(t-\frac{v X}{c^{2}}\right)}$,

which yields just a re-scaling of the position measured by the moving observer expressed by the standard Lorentz transformation for the first spatial coordinate [6].

By following the philosophy of Rueda's and Haisch's interpretation of the inertial mass as an effect of the electromagnetic quantum vacuum [7], in which the presence of a particle with a volume $V_{0}$ expels from the vacuum energy within this volume exactly the same amount of energy, as is particle's internal energy (equivalent to its rest mass). In a similar way in our approach of Quantum Relativity, each material particle is associated with fluctuations of quantum vacuum, which determine a diminishing of the energy density of space. In Quantum Relativity, the energy $E$ of a given physical object with mass $m$ and volume $V$ is the structured energy of quantum vacuum. The amount of energy $E$ and mass $m$ is proportional to the diminished energy density of quantum vacuum $\rho_{\mathrm{qvE}}$ at the center of a given physical object:

$E=m c^{2}=\left(\rho_{\mathrm{PE}}-\rho_{\mathrm{qvE}}\right) V$.

Formula (7) is derived from the formula

$\rho_{\mathrm{qvE}}=\rho_{\mathrm{PE}}-\frac{m c^{2}}{V}$,

ISSN 2071-0194. Ukr. J. Phys. 2018. Vol. 63, No. 7 
where $\rho_{\mathrm{qvE}}$ is the energy density of quantum vacuum inside the physical object, $\rho_{\mathrm{PE}}$ is the Planck energy density [given by (1)], and $V$ is the volume of the physical object.

From formula (8), one can get the following formula regarding the mass of the physical object:

$m=\frac{\left(\rho_{\mathrm{PE}}-\rho_{\mathrm{qvE}}\right) V}{c^{2}}$.

According to relation (9), there is a fundamental symmetry between the property of mass and the changes of the quantum vacuum energy density [8]. From (9), one can get $(7)$, by multiplying the relation by $c^{2}$.

Moreover, in a given volume $V$ of the universal space, the total sum of the different forms of energies (due to the different physical interactions and fields) tends to be constant. This means that, on the basis of the conservation of energy, the average value of the quantum vacuum energy density (8) - intended as a unified field governing the processes taking place in the micro- and macroworlds - is constant in the universe.

Relations (7)-(9) imply that there is an intrinsic relation between the mass $m$ and the quantum vacuum energy density in the sense that the amount of the quantum vacuum energy, which is forming the physical object, its inertial mass $m_{i}$, and its gravitational mass $m_{g}$ are all proportional to the energy $E$ of a given physical object and so to the diminished energy density of quantum vacuum inside of the physical object:

$\frac{E}{c^{2}}=m=m_{i}=m_{g}=\frac{\left(\rho_{\mathrm{PE}}-\rho_{\mathrm{qvE}}\right) V}{c^{2}}$.

On the basis of the equivalence between the mass of a material object and the energy of quantum vacuum, established by Eq. (7), one can consider that, when a material object moves, its kinetic energy is absorbed from vacuum. In other words, the energy taken by a particle from vacuum can be also acquired as the kinetic energy. This means that a relativistic physical object is getting its relativistic energy and relativistic mass by absorbing the additional energy from quantum vacuum. This means that a relativistic physical object additionally diminishes the energy density of quantum vacuum at its center according to the relation

$\rho_{\mathrm{qvE}}=\rho_{\mathrm{PE}}-\frac{m_{0} c^{2}-\frac{m_{0} v^{2}}{2}}{V}$,

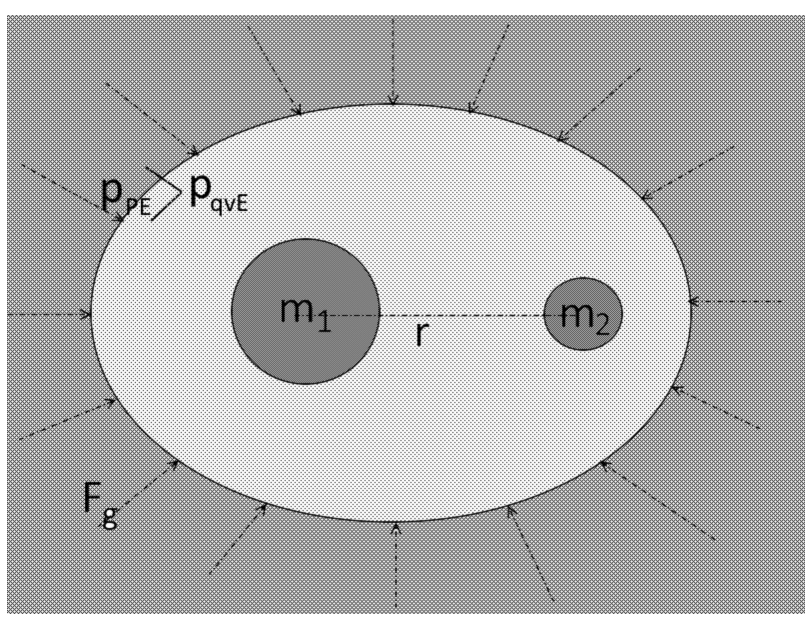

Fig. 1. Gravity as the force based on the difference between the outer energy density of quantum vacuum $\rho_{\mathrm{PE}}$ and the inner energy density of quantum vacuum $\rho_{\mathrm{qvE}}$

where $m_{0}$ is the rest mass of the object and

$v=\sqrt{c^{2}-\frac{\left(\rho_{\mathrm{PE}}-\rho_{\mathrm{qvE}}\right)^{2} V^{2}}{m^{2} c^{2}}}$

is the velocity of the relativistic physical object, which is linked with the variable quantum vacuum energy density and, as one can easily check, is in agreement with the special relativistic prediction that the energy of a physical object diverges in the limit $v \rightarrow c$.

Furthermore, on the basis of the fundamental equation (9) expressing the origin of mass from opportune changes of the quantum vacuum energy density, the well-known Newton gravity formula between two masses $m_{1}$ and $m_{2}$ may be conveniently written as

$F_{g}=\frac{\left(\rho_{\mathrm{PE}}-\rho_{q v E 1}\right) V_{1}\left(\rho_{\mathrm{PE}}-\rho_{q v E 2}\right) V_{2} G}{r^{2} c^{4}}$.

The gravity force is proportional to the masses and inverse proportional to the square of the distance $r$. The bigger the distance $r$, the smaller the difference between the outer energy density of quantum vacuum and the inner energy density of quantum vacuum (see Fig. 1).

The differences between the outer and inner energy densities of quantum vacuum are infinitesimally small. A simple calculation shows that, in the approach based on Eqs. (7)-(10), the gravitational interaction is ruled by infinitesimally small changes of the quantum vacuum energy density. Let us consider 
the values for the quantum vacuum density, which is related to the energy density by $c^{2}$ in the sense that, when we multiply the density of quantum vacuum by $c^{2}$, we get the energy density of quantum vacuum. The Planck density $\rho_{\mathrm{P}}$ of quantum vacuum in the outer intergalactic space is as follows:

$\rho_{\mathrm{P}}=\frac{m_{\mathrm{P}}}{l_{\mathrm{P}}^{3}}$.

Thus,

$\rho_{\mathrm{P}}=5.15500 \times 10^{96} \mathrm{~kg} / \mathrm{m}^{3}$.

At the center of a black hole with the mass $M$ of the Sun and a radius $r$ of $3000 \mathrm{~m}$, the density of quantum vacuum is

$\rho_{q v}=\frac{m_{\mathrm{P}}}{l_{\mathrm{P}}^{3}}-\frac{3 M}{4 \pi r^{3}}$,

namely,

$\rho_{q v}=5.1549999999999999999999999999999 \times$

$\times 10^{96} \mathrm{~kg} / \mathrm{m}^{3}$.

By comparing values (14) and (16), one sees that these values are almost equal: in the calculation of quantity (15), one finds that, after 73 times of number 9 , numbers 824 follow. This shows in other words that infinitesimally small changes of the quantum vacuum energy density are governing gravity. The stability of atoms and subatomic particles requires that the energy density of quantum vacuum has a certain value. Inside of the Schwarzschild radius, the energy density of quantum vacuum goes under that value, which causes atoms and subatomic particles to become unstable. Matter which is structured by the energy of quantum vacuum is disintegrating back into the energy of quantum vacuum.

In Quantum Relativity, light is the vibration of quantum vacuum. That is why light has the same speed in all inertial systems, which all move in quantum vacuum. Light obeys Doppler's effect accordingly to the velocities of the light source and accordingly to the velocities of inertial systems, in which the Doppler effect is measured.

In Quantum Relativity, the rate of clocks depends exclusively on the energy density of quantum vacuum and is valid for all observers. GPS proves that the rate of clocks is valid for all observers regardless they are on the satellite stations, at home, in a train, or in an airplane. Quantum Relativity is abolishing the time as the fourth physical coordinate of space. Time is merely a numerical order of material changes in quantum vacuum and has exclusively mathematical existence. When the numerical order of material changes is measured from the side of an observer, the duration is entering the existence. There is no duration without the measurement.

In this view, no signal can move in the physical time dimension to the present moment. Every signal can move only in space, means in quantum vacuum. CMBR (cosmic microwave background radiation) cannot come from some remote physical past, because such a past does not exist. This is in accord with the vision of Albert Einstein: "Time and space are modes by which we think and not conditions in which we live. ...there is something essential about the NOW which is just outside the realm of science. People like us, who believe in physics, know that the distinction between the past, present and future is only a stubbornly persistent illusion. Time has no independent existence apart from the order of events by which we measure it". According to the Quantum Relativity, CMBR is the characteristic radiation of quantum vacuum, which is the fundamental energy field of the entire universe. In quantum vacuum, it is always NOW. The linear time "pastpresent-future" has only the psychological existence, through which an observer is experiencing the run of physical changes in quantum vacuum [8].

Quantum Relativity is built on the bijective epistemology [9], which sees a given physical object inseparably related to the space (quantum vacuum), in which the physical object exists. In this view, the region of quantum vacuum with a diminished energy density, in which a given physical object exists, is intrinsically connected with the physical object. We cannot approach them separately. The region of quantum vacuum is moving with the physical object. If the object is rotating, the region is rotating as well. In this view, the region of quantum vacuum around the Earth is moving with the Earth. That is why the Michelson-Morley experiment gave the negative result. The Sun also rotates the surrounding quantum vacuum, giving rise to what we call "dragging effect". The dragging effect is the physical origin of the precession of planet. On the basis of Eqs. (7)(9), we have developed a mathematical model for the 
precession of planets, which gave exactly the same result as the calculations by Albert Einstein.

In this paper, our purpose is to put in evidence in what sense the approach of Quantum Relativity provides an improvement of general relativity and quantum theory. In Section 2, we will examine the main results regarding quantum mechanics, which follow from the RS processes of creation/annihilation of the $3 \mathrm{D}$ quantum vacuum. In Section 3, we will analyze the main results regarding special relativity and general relativity. Finally, in Section 4, we will show how this approach determines the suggestive unifying perspectives of quantum mechanics and general relativity, as well as of the electroweak regime of the Standard Model.

\section{Quantum Relativity as an Improvement of Quantum Theory}

Quantum physics, in its modern formulation, has about 90 years. Although it has created many interpretative problems about the description of subatomic processes, its predictions have been confirmed with a incredibly high precision. As regards the geometry of the physical world, quantum physics yields more spread scenarios than those offered by every previous physical theory, showing in particular that the subatomic particles are non-locally connected and can be in entangled states. While in the Copenhagen interpretation owed to Bohr, Heisenberg, and their followers, the non-locality is an unexpected host, which lies behind the purely probabilistic interpretation of the wave function and the mechanism of "casuality" associated with it, on the basis of the experimental results of many authors (such as those by A. Aspect and A. Zeilinger, just to name a few), one has to acknowledge that the non-locality is the ultimate visiting card of the geometry of quantum physics and constitutes an essential property, which is at the basis of the behavior of subatomic particles and the geometry of the quantum world $[10,11]$. In the light of the existence of non-local correlations in the subatomic world, the following questions become natural: what is really a subatomic particle such as an electron? What does it determine the behavior of a subatomic particle? What is the real ultimate background of subatomic processes? Despite the "pacific coexistence" between relativity and quantum physics expressed by the fact that the non-local correlations characterizing the behavior of subatomic particles do

ISSN 2071-0194. Ukr. J. Phys. 2018. Vol. 63, No. 7 not transport energy, and thus they do not violate the laws of relativity, there is furthermore the fundamental problem of building a dynamic model of the space-time background, in which the quantum processes also find a place, in other words of developing a quantum geometrodynamics.

The Quantum Relativity faces the question regarding the non-locality as an essential ultimate visiting card of quantum processes in a direct way by introducing a $3 \mathrm{D}$ non-local quantum vacuum as the fundamental background. Quantum Relativity provides an improvement and a completion of quantum theory in the sense that the behavior of an electron can be seen as the effect of the more elementary processes of formation and dissolving of quanta of the $3 \mathrm{D}$ quantum vacuum. Here, the key point is to introduce Bohm's quantum potential into a $3 \mathrm{D}$ isotropic quantum vacuum characterized by the Planckian metric and defined by elementary processes of creation/annihilation of quanta corresponding to Chiatti's and Licata's transactions.

In a series of recent papers [12, 13], Chiatti and Licata proposed the idea of that, in the physical universe, the ontologically primary events are the creation and annihilation of an elementary quantum, of an actualized transaction, and they correspond to a peculiar reduction of the state vector (which is constituted of interaction vertices, in which real elementary particles are created or destroyed). In the epistemological affinity with Chiatti's and Licata's transactional approach, the Quantum Relativity model postulates that the appearance of baryonic matter derives from an opportune excited state of the 3D quantum vacuum defined by opportune changes of the quantum vacuum energy density and corresponding to the specific reduction-state (RS) processes of creation/annihilation of quanta [4]. The excited state of quantum vacuum corresponding to the appearance of a material particle of mass $m$ is defined (in the center of that particle) by the energy density (8) (and thus by a diminishing with respect to the Planck energy density characterizing the ground state), and its evolution is determined by opportune $\mathbf{R S}$ processes of creation/annihilation of quanta described by a wave function $C=\left(\begin{array}{l}\Psi \\ \phi\end{array}\right)$

with two components satisfying a time-symmetric extension of the Klein-Gordon quantum relativistic 
equation

$\left(\begin{array}{cc}H & 0 \\ 0 & -H\end{array}\right) C=0$

where $H=\left(-\hbar^{2} \partial^{\mu} \partial_{\mu}+\frac{V^{2}}{c^{2}}\left(\Delta \rho_{\mathrm{qvE}}\right)^{2}\right)$ and $\Delta \rho_{\mathrm{qvE}}=$ $=\left(\rho_{\mathrm{PE}}-\rho_{\mathrm{qvE}}\right)$ is a change of the quantum vacuum energy density. Equation (17) corresponds to the following two equations:

$\left(-\hbar^{2} \partial^{\mu} \partial_{\mu}+\frac{V^{2}}{c^{2}}\left(\Delta \rho_{\mathrm{qvE}}\right)^{2}\right) \psi_{Q, i}(x)=0$

for creation events and

$\left(\hbar^{2} \partial^{\mu} \partial_{\mu}-\frac{V^{2}}{c^{2}}\left(\Delta \rho_{\mathrm{qvE}}\right)^{2}\right) \phi_{Q, i}(x)=0$

for destruction events.

The virtual particles-antiparticles corresponding to the RS processes of creation/annihilation of the 3D quantum vacuum give rise to a total zero spin, thus constituting an organized Bose ensemble analogous to the superfluid helium [14]. As a consequence, in agreement with a recent model proposed by Sbitnev in a series of recent papers [15-18], in which the physical vacuum is seen as a superfluid medium and contains pairs of particles-antiparticles, which make up a Bose-Einstein condensate, Quantum Relativity implies that, in presence of ordinary baryonic matter, the $3 \mathrm{D}$ quantum vacuum - and thus the fundamental background, which rules the behavior of subatomic particles - physically acts as a superfluid medium and thus can be characterized by the following Einstein energy-momentum tensor:

$T^{\mu \nu}=(\varepsilon+p) u^{\mu} u^{\nu}+p \eta^{\mu \nu}$,

where $\varepsilon$ and $p$ are functions per unit volume expressed in units of pressure, and the metric tensor $\eta^{\mu \nu}$ has the spacelike signature $(-,+,+,+)$. From the energy-momentum tensor (20), one obtains the following conservation law:

$\partial_{\mu}\left(V T^{\mu \nu} / n\right)=0$,

where $n$ is the number of the RS processes of virtual subparticles characterizing the vacuum medium. Hence, by following the philosophy that underlines Sbitnev's hydrodynamic picture provided in [18], Eqs. (20)-(21) yield the first Fick's law in the relativistic limit in the form

$j_{\mu}=-\frac{D}{c^{2}} \partial_{\mu}\left(\Delta \rho_{\mathrm{qvE}}\right)$, where the diffusion coefficient $D$ - having the dimension of length $h^{2} /$ time - can be seen as a result of the scattering of the subparticles of the RS processes characterizing the vacuum in a given volume $V$ and can be expressed as

$D=\frac{\hbar c^{2} n}{2 \Delta \rho_{\mathrm{qvE}} V}$.

As a consequence of the motion of virtual particles corresponding to the elementary fluctuations of the quantum vacuum energy density, space-time is filled with virtual radiation with frequency $\omega=\frac{c^{2}}{D}$, namely

$\omega=\frac{2 \Delta \rho_{\mathrm{qvE}} V}{\hbar n}$.

In the light of relations (23)-(24), we can say that each elementary fluctuation of the quantum vacuum energy density in a given volume produces an oscillation of the vacuum at a peculiar frequency. This means that each material object given by mass (9) corresponds to oscillations of the vacuum given by relation (24).

The total effect of the motion of virtual particles produced by the RS processes characterizing a given region - in correspondence to changes of the quantum vacuum energy density - is to generate a dragging pushing effect of the 3D quantum vacuum. In particular, one may describe the pushing effect of a region of volume $V$ of the quantum vacuum at a given point at a distance $R$ from the center of that volume by defining a velocity of the $3 \mathrm{D}$ quantum vacuum on the basis of the relation

$v_{q v}=\frac{2 \Delta \rho_{\mathrm{qvE}} V}{\hbar n} R$.

The quantum vacuum velocity (25) is defined with respect to the special rest frame of the Euclid space associated with the quantum vacuum energy density (1). In other words, the rest frame corresponding to the Planck energy density is a special frame, in which the quantum vacuum velocity (25) is zero, all material objects (and thus all variations of the energy density of quantum vacuum) correspond to regions of the quantum vacuum endowed with velocity (25) with respect to this special frame. We will return to the important role of the velocity of quantum vacuum (25) later, in Section 3, in the treatment of relativity.

Let us now see, in mathematical details, in what sense the non-locality is embedded in Quantum Relativity as the ultimate visiting card of processes. By

ISSN 2071-0194. Ukr. J. Phys. 2018. Vol. 63, No. 7 
considering the fundamental equation ruling the $\mathbf{R S}$ processes, namely the Klein-Gordon equation (17), and decomposing the real and imaginary parts of this equation after writing the two components of the wave function in the polar form

$\psi_{Q, i}=\left|\psi_{Q, i}\right| \exp \left(\frac{i S_{Q, i}^{\psi}}{\hbar}\right)$

$\phi_{Q, i}=\left|\phi_{Q, i}\right| \exp \left(\frac{i S_{Q, i}^{\phi}}{\hbar}\right)$,

one obtains a couple of quantum Hamilton-Jacobi equations for the real part that, by imposing the requirement that they are Poincarè invariant and have the correct non-relativistic limit, take the following form:

$\partial_{\mu}\left(\begin{array}{c}S_{Q, i}^{\psi} \\ S_{Q, i}^{\phi}\end{array}\right) \partial^{\mu}\left(\begin{array}{c}S_{Q, i}^{\psi} \\ S_{Q, i}^{\phi}\end{array}\right)=\frac{V^{2}}{c^{2}}\left(\Delta \rho_{\mathrm{qvE}}\right)^{2} \exp \left(\begin{array}{c}Q_{Q, i}^{\psi} \\ -Q_{Q, i}^{\phi}\end{array}\right)$,

while the imaginary part gives the continuity equation

$\partial_{\mu}\left(\sigma \partial^{\mu}\left(\begin{array}{c}S_{Q, i}^{\psi} \\ S_{Q, i}^{\phi}\end{array}\right)\right)=0$,

where $\sigma$ is the ensemble of particles associated with the wave function under consideration and

$Q_{Q, i}=\frac{\hbar^{2} c^{2}}{V^{2}\left(\Delta \rho_{\mathrm{qvE}}\right)^{2}}\left(\begin{array}{c}\frac{\left(\nabla^{2}-\frac{1}{c^{2}} \frac{\partial^{2}}{\partial t^{2}}\right)\left|\psi_{Q, i}\right|}{\left|\psi_{Q, i}\right|} \\ -\frac{\left(\nabla^{2}-\frac{1}{c^{2}} \frac{\partial^{2}}{\partial t^{2}}\right)\left|\phi_{Q, i}\right|}{\left|\phi_{Q, i}\right|}\end{array}\right)$

is the quantum potential of the vacuum. The nonlocality characterizing subatomic processes emerges ultimately from the quantum potential of the vacuum (30), which represents the fundamental entity, which guides the occurring of the processes of creation or annihilation events in space. As a consequence of the primary physical reality of the processes of creation and annihilation and of the non-local action of the quantum potential and in analogy to the epistemology of a recent model proposed by Licata and Chiatti, where the quantum jumps are processes of entry and exit from the usual temporal domain to a timeless vacuum [19], the behavior of the matter in the universe in Quantum Relativity can be seen as an undivided network of $\mathbf{R S}$ processes that take place in

ISSN 2071-0194. Ukr. J. Phys. 2018. Vol. 63, No. 7 a $3 \mathrm{D}$ timeless quantum vacuum, and time has not a primary physical reality but exists merely as a mathematical parameter measuring the dynamics of the particle under consideration.

As regards the RS processes of creation, the quantum potential associated with the virtual particles of the RS processes of the 3D quantum vacuum may be written as

$Q=V \frac{p_{1}+p_{2}}{n}=$

$=-\frac{\hbar^{2} c^{2} n^{2}}{4 \Delta \rho_{\mathrm{qvE}}^{2} V^{2}}\left[\nabla^{2} \Delta \rho_{\mathrm{qvE}}-\frac{1}{c^{2}} \frac{\partial^{2}}{\partial t^{2}} \Delta \rho_{\mathrm{qvE}}\right]+$

$+\frac{\hbar^{2} c^{2} n^{2}}{8 \Delta \rho_{\mathrm{qvE}}^{3} V^{2}}\left[\left(\nabla \Delta \rho_{\mathrm{qvE}}\right)^{2}-\frac{1}{c^{2}}\left(\frac{\partial}{\partial t} \Delta \rho_{\mathrm{qvE}}\right)^{2}\right]$,

where $n$ is the number of virtual particlesantiparticles of the RS processes in the volume $V$ of the $3 \mathrm{D}$ quantum vacuum under consideration [20]. Equation (31) shows that the quantum potential of the vacuum describes the geometry via the pressures $p_{1}$ and $p_{2}$ that arise by the collisions between the virtual particles-antiparticles populating the vacuum and corresponding to the RS processes. In this picture, the quantum potential of the vacuum (31) may be considered as the real origin of the quantum effects. This means that, according to the model of the Quantum Relativity, the quantum potential of ordinary quantum mechanics (that is $Q=-\frac{\hbar^{2}}{2 m} \frac{\nabla^{2} R}{R}$ for a one-body system in the non-relativistic domain) can be considered a consequence of the more fundamental quantum potential of the quantum vacuum (31).

In the Quantum Relativity model presented here, the evolution of each subatomic particle (such as an electron in the double-slit interference) emerges from the opportune elementary $\mathbf{R S}$ processes of creation/annihilation of quanta guided by the quantum potential of the vacuum. The behavior of a subatomic particle, as described by Bohm's approach to quantum mechanics, can thus be seen as a result of the more fundamental evolution of fluctuations of the quantum vacuum energy density corresponding to elementary RS processes of creation/annihilation of quanta. Because of its origin from the elementary fluctuations of the quantum vacuum energy density, an electron can be therefore associated with appropriate waves of the vacuum, which guide it in the different regions of the $3 \mathrm{D}$ quantum vacuum 
through the action of the quantum potential of the vacuum [21].

In particular, the non-local correlations characterizing the quantum domain can be seen as effects deriving from the general Bell length of the $3 \mathrm{D}$ quantum vacuum

$$
\begin{aligned}
& L_{\text {quantum }}= \\
& =\frac{c^{2} \hbar}{\left.\sqrt{\frac{V}{n}\left[-\left(\nabla \Delta \rho_{\mathrm{qvE}}\right)^{2}+\frac{1}{c^{2}}\left(\frac{\partial}{\partial t} \Delta \rho_{\mathrm{qvE}}\right)^{2}-\right.}-\Delta \rho_{\mathrm{qvE}}\left(\nabla^{2} \Delta \rho_{\mathrm{qvE}}-\frac{1}{c^{2}} \frac{\partial^{2}}{\partial t^{2}} \Delta \rho_{\mathrm{qvE}}\right)\right]}
\end{aligned}
$$

which measures the degree of non-local correlation characterizing this background. The quantum length (32) is the ultimate parameter that quantitatively indicates that, at a fundamental level, the $3 \mathrm{D}$ quantum vacuum defined by $\mathbf{R S}$ processes of creation/annihilation of virtual particles-antiparticles corresponding to fluctuations of the quantum vacuum energy density is a non-local and timeless manifold.

On the basis of some recent research, the Bell length as the ultimate parameter, which quantifies the degree of non-local correlations in a quantum system, implies that the maximum delocalization of a quantum system is 1 [22]. As a consequence of this, one obtains the following simple relation satisfied by the number of virtual particles-antiparticles of the RS processes of the 3D quantum vacuum under the condition of maximum entanglement and the maximum grade of non-locality and delocalization in a quantum system having the mass $m=\frac{\Delta \rho_{\mathrm{qvE}} V}{c^{2} n}$ produced by the fluctuations of the quantum vacuum energy density corresponding to the same RS processes:

$$
n^{1 / 2}=\frac{2 \Delta \rho_{\mathrm{qvE}} V^{1 / 2}}{\sqrt{\left[\begin{array}{l}
-\left(\nabla \Delta \rho_{\mathrm{qvE}}\right)^{2}+\frac{1}{c^{2}}\left(\frac{\partial}{\partial t} \Delta \rho_{\mathrm{qvE}}\right)^{2}- \\
-\Delta \rho_{\mathrm{qvE}}\left(\nabla^{2} \Delta \rho_{\mathrm{qvE}}-\frac{1}{c^{2}} \frac{\partial^{2}}{\partial t^{2}} \Delta \rho_{\mathrm{qvE}}\right)
\end{array}\right]}} .
$$

In virtue of the fundamental features of the 3D quantum vacuum in Quantum Relativity, one can throw new light as regards the problem of the existence, in quantum physics, of different descriptive levels of physical reality, whether formal analogies exist between them or there is a deeper meaning. In fact, by using a Bohmian terminology, the reality constituted by the RS processes and their evolution through the non-local action of the quantum potential of the vacuum can be defined as the background, from whose differentiation the foreground constituted by the events of a given subatomic particle or system (governed by the well-known laws of quantum theory) emerges. In this picture, the ordinary spacetime has no physical reality of its own, but it is, so to speak, materialized by RS processes. In an analogous way, the background associated with the de Broglie-Bohm pilot-wave theory but also of Bohm's implicate order and Hiley's pre-space seen as manifolds derived from this more fundamental arena represented by the three-dimensional nonlocal timeless quantum vacuum are someway "materialized" by RS processes. The philosophy of this model as regards the link between our level of physical reality (regarding measurement processes), the implicate order, and the 3D timeless non-local quantum vacuum actually follows someway the program that Bohm had already sketched out, when he had studied the relationship between implicate and explicate orders. At the same time, it allows us to go beyond it, suggesting a possible deeper origin and explanation of Bohm's implicate order. One can say that, since each subatomic particle (such as an electron in the famous double-slit interference experiment) is indeed the evolution of $\mathbf{R S}$ processes of creation/annihilation of quanta corresponding to elementary fluctuations of the quantum vacuum energy density, there is an equivalence between the 3D timeless non-local quantum vacuum and the space-time background of the subatomic particle under consideration (in this sense, there is a formal analogy between the 3D timeless non-local quantum vacuum and the usual background of quantum theory). Moreover, the Quantum Relativity allows us to suggest a deeper meaning of the relationships between the different descriptive levels of physical reality in the sense that, with the introduction of a 3D timeless non-local quantum vacuum defined by $\mathbf{R S}$ processes of creation/annihilation of quanta corresponding to elementary fluctuations of the quantum vacuum energy density, the ordinary quantum mechanics emerges directly from the $3 \mathrm{D}$ timeless non-local quantum vacuum [21].

Finally, the Bell length of the vacuum introduces interesting perspectives as regards a new re-reading of the well-known Bell inequalities investigating the correlations between two particles that share a quantum 
state $[23-25]$. As regards the quantum dynamics of a general two-qubit state, with vanishing total angular momentum projections, given by the equation

$|\psi\rangle=\cos \frac{\vartheta}{2}|\uparrow \downarrow\rangle+e^{i \varphi} \sin \frac{\vartheta}{2}|\downarrow \uparrow\rangle$,

where $|\uparrow \downarrow\rangle$ corresponds to the state of the system, when the first qubit is in the "up" state and the second qubit is in the "down" state, while $|\downarrow \uparrow\rangle$ corresponds to the state of the system, when the first qubit is in the "down" state and the second qubit is in the "up" state, the quantum potential in the Bohmian framework is

$Q=\frac{\left(\hat{M}_{1}^{2}+\hat{M}_{2}^{2},\right) R}{2 I R}$.

Here, $\mathbf{M}_{1}=i \hat{\mathbf{M}}_{1} S$ and $\mathbf{M}_{2}=i \hat{\mathbf{M}}_{2} S$ are the angular momenta of two qubits 1 and 2, respectively, $S$ being the phase of the wave function of the system. The grade of non-locality in this system may be characterized by considering the following Bell length:

$L_{\text {quantum }}=\frac{1}{\sqrt{\frac{\left(\left(\hat{\mathbf{M}}_{1}^{2}+\hat{\mathbf{M}}_{2}^{2}\right) R\right)}{R}}}$.

In the approach of Quantum Relativity, since each quantum system is the evolution of opportune RS processes of creation/annihilation of quanta corresponding to elementary fluctuations of the 3D quantum vacuum, the Bell length (36) regarding a system of two qubits in the entangled state (34) can be derived from a more fundamental Bell length of the vacuum (32). More precisely, one can say that the behavior of a system of two qubits in the entangled state (34) described by the quantum laws is ultimately determined by RS processes of creation/annihilation of quanta corresponding to elementary fluctuations of the $3 \mathrm{D}$ quantum vacuum on the basis of the equation

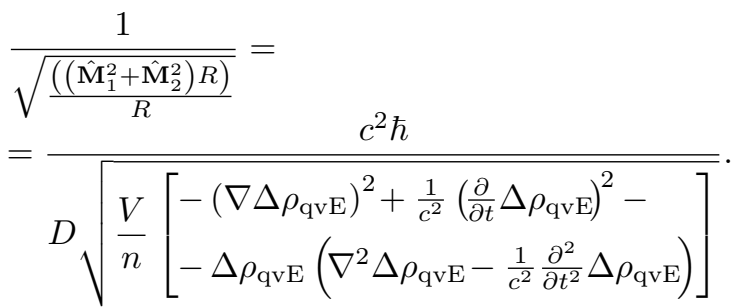

Now, in the light of these considerations, by following the philosophy underlying the approach of Hall's correlation distance developed in [26], we can suggest in our model of Quantum Relativity that the standard Bell inequality in the form derived by Clauser, Horne, Shimony, and Holt (CHSH),

$\mathrm{CHSH} \equiv\langle A B\rangle+\left\langle A B^{\prime}\right\rangle+\left\langle A^{\prime} B\right\rangle-\left\langle A^{\prime} B^{\prime}\right\rangle \leqslant 2$,

may be generalized as

$\langle A B\rangle+\left\langle A B^{\prime}\right\rangle+\left\langle A^{\prime} B\right\rangle-\left\langle A^{\prime} B^{\prime}\right\rangle \leqslant \frac{4}{2-L_{\text {quantum }}^{\text {max }}}$,

where $L_{\text {quantum }}^{\max }=1$ is the maximum value of the Bell length of the vacuum that is directly determined by opportune fluctuations of the quantum vacuum energy density on the basis of Eq. (32). By substituting Eq. (32) into Eq. (39), we obtain

$$
\begin{aligned}
& \langle A B\rangle+\left\langle A B^{\prime}\right\rangle+\left\langle A^{\prime} B\right\rangle-\left\langle A^{\prime} B^{\prime}\right\rangle \leqslant
\end{aligned}
$$

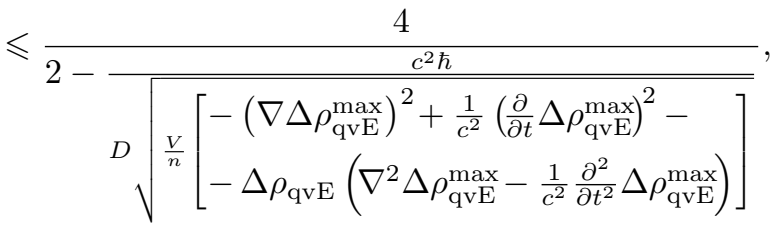

where $\Delta \rho_{\mathrm{qvE}}^{\max }$ are the opportune quantum vacuum energy fluctuations determining the maximum degree of non-local correlations in a quantum system. Therefore on the basis of the Bell length of the vacuum (32), a quantum vacuum inequality can be introduced in the form

$\mathrm{CHSH}_{\text {vacuum }} \leqslant 0$,

where

$$
\begin{aligned}
& \mathrm{CHSH}_{\text {vacuum }}=\langle A B\rangle+\left\langle A B^{\prime}\right\rangle+\left\langle A^{\prime} B\right\rangle-\left\langle A^{\prime} B^{\prime}\right\rangle-
\end{aligned}
$$

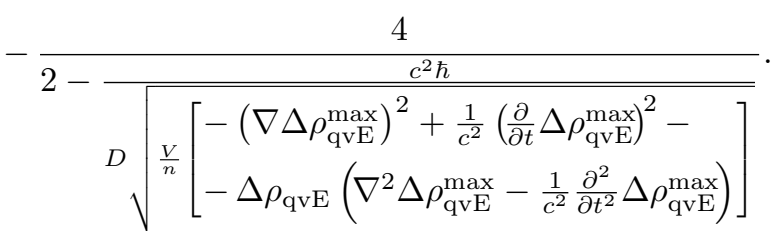

In the Quantum Relativity approach, the violation of the quantum vacuum inequality (41) essentially coincides with the one of the standard $\mathrm{CHSH}$ inequality. However, the measurements that maximize the violation of $\mathrm{CHSH}$ are not the ones, which give the

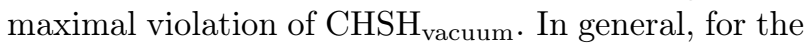


standard Bell-CHSH scenario, the violation of the standard inequality (38) is a necessary but not sufficient condition for the violation of (41). The advantage of the quantum vacuum inequality (41) lies in the fact that the results depend causally on the Bell length of the vacuum, which emerges directly as the ultimate parameter determining, at the fundamental level of the 3D quantum vacuum defined by RS processes of creation/annihilation of quanta corresponding to elementary fluctuations of the quantum vacuum energy density, the correlation degree in quantum systems.

\section{About the Advances of Quantum Relativity Regarding Special Relativity and General Relativity}

In Quantum Relativity, time has only a mathematical meaning, namely, time is a numerical order of changes running in space. Time is not the $4^{\text {th }}$ dimension of space. In Quantum Relativity, light has the same velocity in all inertial systems, because the source of light and all inertial systems, in which the light is observed, are moving in the same space (3D quantum vacuum); a photon is the wave of space (3D quantum vacuum), in which the source of light and all inertial systems move. Light behaves itself accordingly to the Doppler law. All phenomena of special relativity are in Quantum Relativity described in a 3D space. There is no "time dilation" and "length contraction," which are only the mathematical tools in the Einstein theory to preserve the light speed constancy.

In Quantum Relativity, the rate of clocks is valid for all observers. Quantum Relativity abolishes "internal observer", "external observer," and "coordinate time" of special relativity. In Quantum Relativity, the rate of clocks in all inertial systems is valid for all observers. GPS proves that. The Special Relativity effect of a slower rate of clocks on the satellites and the General Relativity effect of a faster rate of clocks on the satellites have, in Quantum Relativity, the origin in the variable energy density of quantum vacuum. Satellites are moving with higher velocity than the Earth surface. Because of this, they additionally absorb the energy of quantum vacuum, which actually is the kinetic energy of satellites. The additional absorption of the quantum vacuum energy in the form of a kinetic energy causes the additional diminishing of the energy density of quantum vacuum inside of a satellite, which causes that the rate of clocks is slower. As for the General Relativity effect related to the motion in the direction away from the Earth in the space, the energy density of quantum vacuum is increasing, and the rate of clocks is faster. The variable rate of clocks in all inertial systems is valid for all observers.

In Quantum Relativity, the light speed is constant in the regions of the universal space with unchanged energy density of quantum vacuum. Minimal changes of the quantum vacuum energy density cause minimal changes of the light speed in accord with classical physics' discovery, namely, the motion speed of a signal is increasing with the density of the medium, in which signal is moving. The Shapiro experiment proves that light diminishes minimally the speed in stronger gravity, where the energy density of quantum vacuum is lower. General Relativity describes the Shapiro experiment as "gravitational time dilation", which means that, in a stronger gravity field, the $4^{\text {th }}$ time coordinate is dilating, getting longer. Respectively, the time of the light motion from point $A$ to point $B$ increases. This interpretation is not realistic. Quantum Relativity is abolishing the gravitational time dilation, which is purely a mathematical model and has no correspondence in the physical world.

In Quantum Relativity, the relativistic decay of muons [27] has origin in the diminished energy density of quantum vacuum. Muons are moving toward the Earth with high speed, which increases their kinetic energy and additionally diminishes the energy density of quantum vacuum. The diminished energy density of quantum vacuum causes that all physical changes, including muons' decay, are slower. In Quantum Relativity, there is no "time dilation," as it is in Special Relativity, in the sense that time dilates as the physical reality, in which material changes run. In Quantum Relativity time is merely numerical order of the material changes. When we measure this numerical order of material changes with the clocks, we get the duration. Muons move in quantum vacuum, where it is always NOW. Their high velocity additionally diminishes the energy density of quantum vacuum, which causes that the decay of muons is getting slower and is valid for all observers. The rate of clocks in Quantum Relativity does not depend on the observer position; it depends only on the energy density of quantum vacuum.

ISSN 2071-0194. Ukr. J. Phys. 2018. Vol. 63, No. 7 
Quantum Relativity fully explains the Sagnac effect, by which a light signal needs more time, when travelling from point $\mathrm{A}$ to point $\mathrm{B}$ in the opposite direction of Earth's rotation. With the rotating Earth, the quantum vacuum area around the Earth is also rotating. As a photon is the wave of quantum vacuum, it needs more time, when moving in the opposite direction of the movement of quantum vacuum.

A detailed analysis of the Sagnac effect, which regards the different velocities of different light signals relative to an interferometer, can be found, for example, in Post's classical paper [28]. The original apparatus utilized by Sagnac in his famous experiment is depicted in Fig. 2: a light source $\mathrm{S}$ generates light signals, which are divided by the half-silvered-mirror HSM into two beams, which follow a clockwise or counter-clockwise path of the same length back to HSM, where they are recombined and detected at a final mirror D. Sagnac's experiment shows that when this interferometer is rotated with angular rate $\boldsymbol{\Omega}$ $\mathrm{rad} / \mathrm{sec}$, a fringe shift $\Delta Z$ given by the relation

$\Delta Z=4 \mathbf{\Omega} \cdot \mathbf{A} /\left(\lambda_{0} c\right)$

is observed. It corresponds, at the lowest order in the vector $\boldsymbol{\Omega}$ providing the direction and the rate of rotation, to a phase difference between the counterrotating beams of

$\Delta \varphi=2 \pi \Delta Z=8 \pi \boldsymbol{\Omega} \cdot \mathbf{A} /\left(\lambda_{0} c\right)$.

In Eqs. (43)-(44), $\lambda_{0}$ is the vacuum wavelength of light, $|\bar{A}|$ is the area enclosed by the circulating light beams, and $\mathbf{A}$ is perpendicular to the plane of an interferometer. The scalar product $\boldsymbol{\Omega} \cdot \mathbf{A}$ indicates that the phase difference between the counterrotating beams is proportional to the cosine of the angle between the axis of rotation and the normal to the optical circuit. Equations (43)-(44) physically mean that when an interferometer is rotated, there are different transit times from a beam-splitter to a beam-splitter for clockwise- and counter-clockwiserotating beams. Moreover, it is of interest to remark that, by making an electromagnetic discussion of the Sagnac effect in the context of a geometric optical theory, Post obtained in [28] that the actually observed fringe shift produced by the clockwise and counterclockwise beams in a moving interferometer with comoving medium, when compared with the stationary

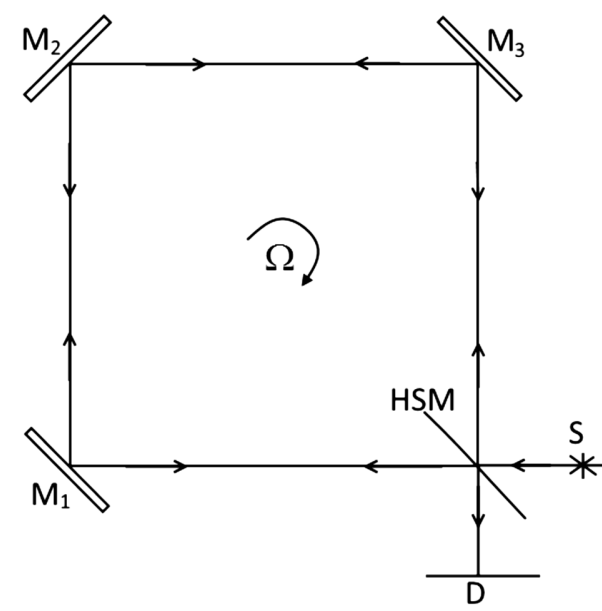

Fig. 2. A Sagnac interferometer

case, can be expressed as

$\Delta Z=\frac{2 c}{\lambda_{0}} \oint n^{2}(1-\alpha) \mathbf{v} \cdot d \mathbf{r}$,

where $n$ is the index of refraction in the stationary medium, $\mathbf{v}$ is the velocity field, $d \mathbf{r}$ is the vector line element, and the equivalence between (45) and (43) may be reproduced, if one sets $\alpha=1-n^{-2}$ and uses the following relation based on the Stokes theorem:

$\oint \mathbf{v} \cdot d \mathbf{r}=\oint \operatorname{curl} \mathbf{v} \cdot d \mathbf{A}=2 \boldsymbol{\Omega} \cdot \mathbf{A}$.

Now, in our approach, the vector $\Omega$, which provides the direction and the rate of rotation of a Sagnac interferometer, derives from the motion of the virtual particles of the RS processes corresponding to elementary fluctuations of the quantum vacuum energy density, namely, it can be associated with the frequencies given by Eq. (24). In other words, the vector $\boldsymbol{\Omega}$ may be expressed as

$\boldsymbol{\Omega}=\frac{2 \Delta \rho_{\mathrm{qvE}} V}{\hbar n} \hat{N}$

where $\hat{N}$ is the unit vector identifying the direction of the vector $\boldsymbol{\Omega}$. It is vector (47) associated with the frequencies of the motion of virtual particles of the RS processes of the quantum vacuum that determines the phase difference between the counterrotating beams in the Sagnac experiment. In other words, one can say that, as a consequence of these elementary fluctuations of the quantum vacuum energy 


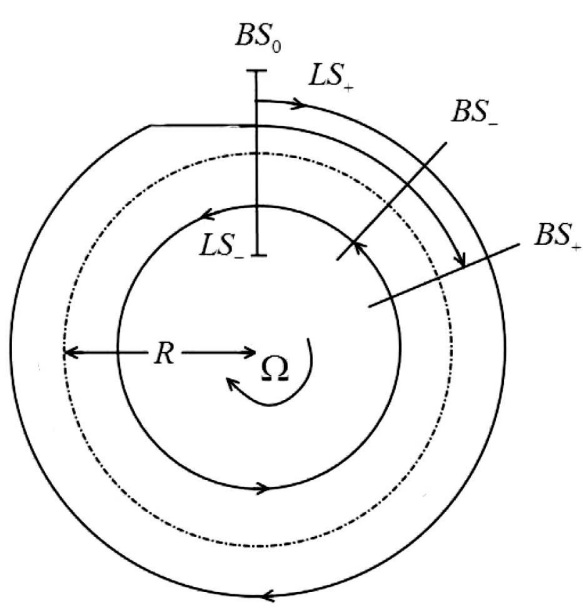

Fig. 3. A circular Sagnac interferometer of radius $R$ rotating with uniform angular velocity $\Omega$ in the clockwise direction [30]

density, when the whole Sagnac apparatus, including the light source and the detector, is rotated, the fringe shift between clockwise- and counter-clockwiserotating beams corresponds, at the lowest order in the angular velocity, to a phase difference between the counter-rotating beams of the form

$\Delta \varphi=16 \pi \frac{\Delta \rho_{\mathrm{qvE}} V}{\hbar n} \hat{N} \cdot \mathbf{A} /\left(\lambda_{0} c\right)$.

On the basis of Eq. (48), we can say that the real origin, the real ultimate visiting card of the phase shift of a Sagnac interferometer, is represented by motion of the virtual particles of the RS processes of the 3D quantum vacuum. Moreover, taking Eq. (25) into account and by introducing the length $L$ of the fiber optic path, Eq. (44) may also be formulated as

$\Delta \varphi=8 \pi \frac{v_{q v}}{L} \hat{N} \cdot \mathbf{A} /\left(\lambda_{0} c\right)$,

where $A=4 L^{2}$ is the area enclosed by the moving light beams. Equation (49) shows that the phase difference between the counter-rotating beams is proportional to the velocity of the quantum vacuum determined by the motion of virtual particles produced in the $\mathbf{R S}$ processes of the region under consideration [29]. Moreover, in a special relativistic picture, the phase difference (49) may be expressed as

$$
\Delta \varphi=8 \pi \frac{v_{q v}}{L} \hat{N} \mathbf{A} /\left(\lambda_{0} c\right)\left[1-\frac{13}{24}\left(\frac{2 \Delta \rho_{\mathrm{qvE}} V L}{\hbar n c}\right)^{2}\right]+
$$

$+O\left(\left(\frac{2 \Delta \rho_{\mathrm{qvE}} V L}{\hbar n c}\right)^{5}\right)$

In view of a comparison with Post's treatment, it is of interest to consider a Sagnac circular interferometer of radius $R$ rotating with uniform angular velocity in the clockwise direction. Here, by following to Field [30], we consider $L S_{+}$and $L S_{-}$co-rotating and counter-rotating light signals, which depart simultaneously from a beam splitter (BS), when it is positioned at $B S_{0}$ (see Fig. 3 ). The signals $L S_{-}$ $\left(L S_{+}\right)$arrive back at BS, when it is in the laboratory frame positions $B S_{-}\left(B S_{+}\right)$. In the laboratory frame, both light signals move with speed $c$. The different arrival times result from different laboratory frame path lengths followed by the signals.

In this case, taking Field's results into account, the relative velocities of the light signals and an interferometer determined by the dragging effect of the quantum vacuum are given by

$c_{r}^{ \pm}=c \mp\left(\frac{2 \Delta \rho_{\mathrm{qvE}} V R}{\hbar n}\right)$

namely

$c_{r}^{ \pm}=c \mp v_{q v}$,

where $c_{r}^{+}\left(c_{r}^{-}\right)$are the velocities of clockwise (counterclockwise) rotating light signals, relative to an adjacent point on the interferometer, in the laboratory system. The times-of-passage of the light signals from a beam-splitter to a beam-splitter in the laboratory system for the counter-rotating signals are

$T_{ \pm}=\frac{2 \pi R}{c \mp\left(\frac{2 \Delta \rho_{\mathrm{qvE}} V R}{\hbar n}\right)}$,

namely

$T_{ \pm}=\frac{2 \pi R}{c \mp v_{q v}}$

In a special relativistic picture, by using the differential Lorentz transformations from the laboratory system into the instantaneous co-moving frame of the beam splitter BS, the phase shift can be expressed as

$\Delta \phi_{\mathrm{SR}}=\frac{16 \pi \Delta \rho_{\mathrm{qvE}} V A}{\lambda_{0} \hbar n c}\left[1+\frac{1}{2}\left(\frac{2 \Delta \rho_{\mathrm{qvE}} V R}{\hbar n c}\right)^{2}\right]+$
$+O\left(\left(\frac{2 \Delta \rho_{\mathrm{qvE}} V R}{\hbar n c}\right)^{5}\right)$, 
namely

$\Delta \phi_{\mathrm{SR}}=\frac{8 \pi v_{q v} A}{\lambda_{0} R c}\left[1+\frac{1}{2}\left(\frac{v_{q v}}{c}\right)^{2}\right]+O\left(\left(\frac{v_{q v}}{c}\right)^{5}\right)$,

where $A=\pi R^{2}$.

Equations (55) and (56) may be considered as the counterparts of Eq. (50) as regards the Sagnac circular interferometer. In other words, the physical significance of Eqs. (55)-(56), on the one hand, and of Eq. (50), on the other hand, is the same. Both for a linear interferometer and for a circular interferometer, the phase difference between the counter-rotating beams (with respect to the stationary state) is determined by the elementary fluctuations of the quantum vacuum energy density, and thus by the velocity of the quantum vacuum, in the area $A$ enclosed by the moving light beams. The only difference between the two cases is as follows: while the area in the linear interferometer depends of the length $L$ of the fiber optic path, it is linked in the circular interferometer with its radius $R$.

As a consequence of the phase difference expressed by Eqs. (55)-(56), in the circular interferometer, the relative velocities of the light signals and the interferometer in the co-rotating systems are:

$T_{ \pm}^{\prime}=\frac{2 \pi R}{\gamma c_{r}^{ \pm}}$

where

$\gamma=\frac{1}{\sqrt{1-\left(\frac{2 \Delta \rho_{\mathrm{qvE}} V R}{\hbar n c}\right)^{2}}}=\frac{1}{\sqrt{1-\left(\frac{v_{q v}}{c}\right)^{2}}}$.

Relations (57) indicate that the signal flight times in the co-rotating frame are determined by the motion of virtual particles of the $\mathbf{R S}$ processes of the $3 \mathrm{D}$ quantum vacuum and turn out to be in agreement with the calculations previously performed by Tartaglia [31]. Moreover, Eqs. (57) show that the relative velocities of the light signals and the circular interferometer transform between the laboratory and co-rotating frames according to the relation

$\left(c_{r}^{ \pm}\right)^{\prime}=\gamma\left[c \mp\left(\frac{2 \Delta \rho_{\mathrm{qvE}} V R}{\hbar n}\right)\right]$,

namely

$\left(c_{r}^{ \pm}\right)^{\prime}=\gamma\left[c \mp\left(v_{q v}\right)\right]$, which allow us to provide a new key of reading the previous results by Klauber [32]. From Eq. (60), it follows also that

$\frac{\left(c_{r}^{+}\right)^{\prime}}{\left(c_{r}^{-}\right)^{\prime}}=\frac{c-\left(\frac{2 \Delta \rho_{\mathrm{qvE}} V R}{\hbar n}\right)}{c+\left(\frac{2 \Delta \rho_{\mathrm{qvE}} V R}{\hbar n},\right)}$

namely

$\frac{\left(c_{r}^{+}\right)^{\prime}}{\left(c_{r}^{-}\right)^{\prime}}=\frac{c-v_{q v}}{c+v_{q v}}$,

which is in agreement with Selleri's inertial transformations

$\left\{\begin{array}{l}x^{\prime}=\frac{x_{0}-v t_{0}}{\sqrt{1-\beta^{2}}}, \\ y^{\prime}=y_{0}, \\ z^{\prime}=z_{0}, \\ t^{\prime}=\sqrt{1-\beta^{2}} t_{0},\end{array}\right.$

which determine an arena of special relativity, in which the temporal coordinate must be clearly considered as a different entity with respect to the spatial coordinates, just because the transformation of clocks' run between the two inertial systems does not depend on the spatial coordinates.

On the light of the treatment of the Sagnac effect based on Eqs. (47)-(62), one can say that the different velocities of clockwise- and counter-clockwiserotating light beams relative to the Sagnac interferometer (both in the cases of the linear and circular interferometers) is really due to the motion of virtual particles of the RS processes of the $3 \mathrm{D}$ quantum vacuum and, therefore, to the velocity of the quantum vacuum. The motion of virtual particles, which arise in the $\mathbf{R S}$ processes of the $3 \mathrm{D}$ quantum vacuum can be considered as the ultimate visiting card, which determines the different velocities of clockwiseand counter-clockwise-rotating light beams relative to the Sagnac interferometer. In virtue of the motion of virtual particles of the RS processes, the quantum vacuum around the Earth is turning with it, and as Eqs. (47)-(62) explicitly demonstrate - this causes that the light signal between two clocks moves with higher velocity in the direction of Earth's rotation and with lower velocity in the opposite direction. A turning quantum vacuum, in which a photon moves, influences its velocity (see Fig. 4). 


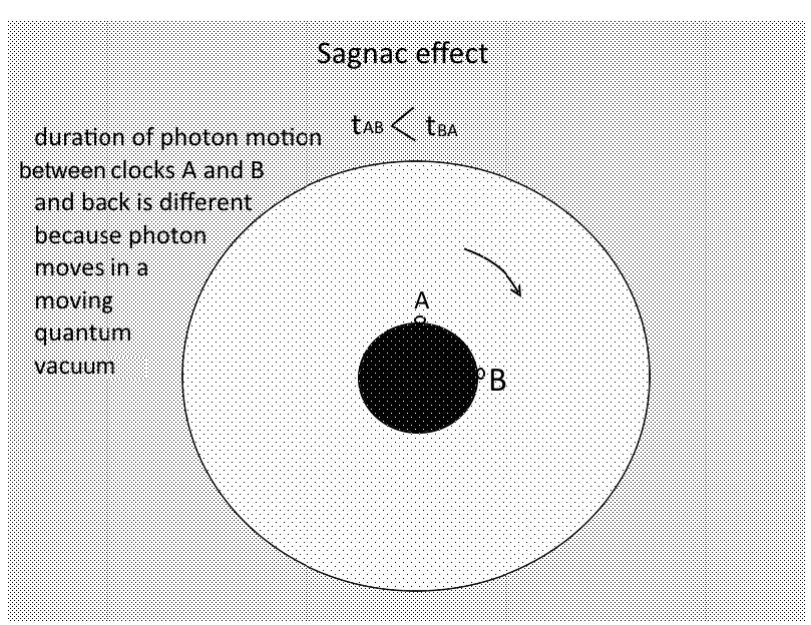

Fig. 4. Sagnac effect by measuring the light signal velocity

It must be emphasized that the above interpretation of the Sagnac effect in terms of fundamental fluctuations of the quantum vacuum energy density turns out to be compatible with the consideration of the Doppler effect between different light signals moving in the different arms of the interferometer. In fact, if $\omega_{0}$ is the light frequency in free space, it gets, as a consequence of the Doppler effect, an increment $\pm \Delta \omega$ depending on along which arm of the interferometer the light spreads, and it is this increment which can be associated with the different velocities of the clockwise and counter-clockwise rotating light signals (52) and, consequently, of their respective times-of-passage from a beam-splitter to a beamsplitter (54). In particular, in the case of the circular interferometer, on the basis of Eqs. (52), the increment $\Delta \omega$ of the frequency depending on along which arm of the interferometer the light spreads is

$\Delta \omega^{ \pm}=\mp \frac{v_{q v}}{\lambda_{0}}$,

$\lambda_{0}$ being the usual wavelength of light in vacuum. According to Eq. (64), the frequency difference between clockwise and counter-clockwise rotating light signals is determined just by the velocity of the quantum vacuum, and thus by fluctuations of the quantum vacuum energy density. The treatment of the Sagnac effect in terms of the Doppler effect determined by a velocity of the quantum vacuum, described by Eq. (64), may be compared to the one of the transmission of signals from rate-adjusted transmitters orbiting on
Global Positioning System (GPS) satellites. On the basis of Ashby's results [33], the frequency jumps arising from satellite orbit adjustments - used by GPS in order to provide the world-wide position and the time determination - allow several relativistic effects, such as just the Sagnac effect, to be explained. In particular, according to Ashby's results, if $f_{0}=10^{23} \mathrm{MHz}$ is the frequency of the satellite transmission, before the rate adjustment is done, after considering the rate adjustment, the received frequency for a receiver fixed on Earth's rotating geoid is

$$
f_{R}=f_{0}\left[1+\frac{2 G M_{E}}{c^{2}}\left(\frac{1}{\alpha}-\frac{1}{r}\right)\right] \frac{\left(1-\mathbf{N} \cdot \mathbf{v}_{R} / c\right)}{\left(1-\mathbf{N} \cdot \mathbf{v}_{j} / c\right)}
$$

where $\alpha$ is the semimajor axis of the orbit of a satellite, $M_{E}$ is Earth's mass, $\mathbf{N}$ is a unit vector in the propagation direction in the local inertial frame, $\mathbf{v}_{R}$ is the velocity of a receiver, $\mathbf{v}_{j}$ is the velocity of the satellite. In our opinion, Ashby's formalism (65) regarding the Doppler effect of signals coming from GPS satellites is compatible with our treatment of the Sagnac effect in the context of the Quantum Relativity approach. What we do here more than Ashby's formalism, in our interpretation of the Sagnac effect, is to suggest that the variation of the frequency of the light depending on along which arm of the interferometer the light spreads, is owed to the motion of virtual particles of the RS processes of the quantum vacuum, associated with the fluctuations of the quantum vacuum energy density, namely by the different values of the velocity of the quantum vacuum depending on along which arm of the interferometer the light spreads, as established by Eq. (64).

Let us examine now the main results regarding the treatment of the gravitational interaction. In this regard, in Quantum Relativity, the curvature of spacetime in General Relativity is only the mathematical description of the energy density of quantum vacuum in a given region of space. This is valid from the micro- to macroscale. The changes and fluctuations of the quantum vacuum energy density generate a curvature of space-time similar to the curvature produced by a "dark energy" density [5, 20], through a quantized metric, characterizing the underlying microscopic geometry of the $3 \mathrm{D}$ quantum vacuum, expressed by the relation

$d \hat{s}^{2}=\hat{g}_{\mu \nu} d x^{\mu} d x^{\nu}$ 
where the (quantum operators) coefficients of the metric are defined (in polar coordinates) as

$$
\begin{aligned}
& \hat{g}_{00}=-1+\hat{h}_{00}, \hat{g}_{11}=1+\hat{h}_{11}, \hat{g}_{11}=1+\hat{h}_{11}, \\
& \hat{g}_{33}=r^{2} \sin ^{2} \vartheta\left(1+\hat{h}_{33}\right), \hat{g}_{\mu \nu}=\hat{h}_{\mu \nu} \text { for } \mu \neq \nu
\end{aligned}
$$

and

$\left\langle\hat{h}_{\mu \nu}\right\rangle=0$

except

$\left\langle\hat{h}_{00}\right\rangle=\frac{8 \pi G}{3}\left(\frac{\Delta \rho_{\mathrm{qvE}}}{c^{2}}+\frac{35 G c^{2}}{2 \pi \hbar^{4} V}\left(\frac{V}{c^{2}} \Delta \rho_{\mathrm{qvE}}^{D E}\right)^{6}\right) r^{2}$,

and

$\left\langle\hat{h}_{11}\right\rangle=\frac{8 \pi G}{3}\left(-\frac{\Delta \rho_{\mathrm{qvE}}}{2 c^{2}}+\frac{35 G c^{2}}{2 \pi \hbar^{4} V}\left(\frac{V}{c^{2}} \Delta \rho_{\mathrm{qvE}}^{D E}\right)^{6}\right) r^{2}$.

In this picture, the dark energy represents itself the structured energy of space on the basis of the equation

$\rho_{D E} \cong \frac{35 G c^{2}}{2 \pi \hbar^{4} V}\left(\frac{V}{c^{2}} \Delta \rho_{\mathrm{qvE}}^{D E}\right)^{6}$

and the variable energy density of space (producing the dark energy) acts as a two-point correlation function according to the relation

$$
\frac{c^{4}}{4 \pi \hbar^{4}}\left(\frac{V}{c^{2}} \Delta \rho_{\mathrm{qvE}}^{D E}\right)^{6} \cong \int_{0}^{\infty} C(s) s d s
$$

where $C(s)$ is the two-point correlation function of the quantum vacuum fluctuations, which depends only on the distance between the two points. In Quantum Relativity on the basis of Eqs. (66)-(70), the $3 \mathrm{D}$ quantum vacuum defined by the quantized metric (66) determined by the changes and fluctuations of the energy density of space can be considered as the ultimate visiting card of general relativity, namely as the fundamental origin of the curvature of space-time characteristic of general relativity.

Finally, the equivalence principle does not need here to be independently postulated but can be seen as a consequence of the RS processes and, thus, the elementary fluctuations of the energy density of the $3 \mathrm{D}$ quantum vacuum. The inertial mass of an object emerges from the interacting fraction of an energy density characterizing the electromagnetic properties of the 3D quantum vacuum defined by the spectral energy density for the zero-point fluctuations

$\rho\left(\Delta \rho_{\mathrm{qvE}}\right)=\frac{4\left(\Delta \rho_{\mathrm{qvE}}\right)^{3} V^{3}}{\hbar^{2} \pi^{2} n^{3} c^{3}}$.

They are determined by the frequencies associated to opportune RS processes of creation/annihilation corresponding to elementary fluctuations of the energy density of space, according to the equation

$m_{i}=\left[4 \frac{V^{4}}{\hbar^{2} \pi^{2} n^{3} c^{5}} \int \eta(\rho)\left(\rho_{p E}-\rho\right)^{3} d \rho\right]$

where $c$ is the speed of light, $\eta(\omega)$ is the spectral factor, interacting with the body, which physically measures the relative strength of the interaction between the oscillations produced by the motions of virtual particles of the RS processes and the massive object, whose interaction acts to oppose the acceleration [2]. Therefore, the quantum vacuum inertia hypothesis suggested by Rueda and Haisch in [7, 3436 ] gets here a new significance: the drag force, which acts as the inertia reaction force of an object that is being forced to accelerate, can be derived from the oscillations associated with the radiation produced by the motions of virtual particles of the RS processes. Namely, it is related to elementary fluctuations of the energy density of the same 3D quantum vacuum. These fundamental quantum vacuum energy density fluctuations lead to the following associated non-relativistic form of the inertia reaction force:

$\mathbf{f}_{*}^{z p}=-m_{i} \mathbf{g}_{\omega}$,

where $\mathbf{g}_{\omega}$ is the acceleration, with which the frequency $\omega$ appears in a local inertial frame $I_{*}$, and the coefficient $m_{i}$ is given by $(72)$. Here, it should be emphasized that the coupling function $\eta(\omega)$ of the inertial mass (72) has different shapes for an electron, a given quark, a composite particle like a proton, a molecule, a homogeneous dust grain, or a homogeneous macroscopic body. However, what appears as the inertial mass, $m_{i}$, to the observer in a local Lorentz frame $I_{*, L}$ corresponds to the passive gravitational mass, $m_{g}$. Thus, we have

$m_{g}=\left[4 \frac{V^{4}}{\hbar^{2} \pi^{2} n^{3} c^{5}} \int \eta(\omega)\left(\rho_{p E}-\rho\right)^{3} d \omega\right]$

[20]. 
4. Unifying Perspectives of Quantum Relativity: From Gravity and the Quantum as Different Aspects of the Same Coin... to the Electroweak Scale and the Interpretation of a Higgs Boson

In addition to the possibility to explain and justify the quantum behavior of subatomic particles at a more fundamental level and to provide a completion and improvement of special relativity and general relativity regarding various aspects (such as the explanation of the Sagnac effect, curvature of space, and dark energy), Quantum Relativity introduces other interesting and relevant unifying perspectives.

Before all, it suggests us to interpret gravity and quantum behavior as two different aspects of a same source, of a same coin. In the spirit of the philosophy that is at the basis of F. Shojai's and A. Shojai's model developed in [37, 38], the quantum behaviors of matter and gravity in Quantum Relativity constitute two different aspects following from the same $3 \mathrm{D}$ timeless non-local quantum vacuum characterized by $\mathbf{R S}$ processes of creation/annihilation corresponding to elementary fluctuations of the quantum vacuum energy density. In this regard, by starting from the Hamilton-Jacobi equation (28) regarding Bohm's version of the Klein-Gordon equation of the generic component of the probability amplitude of the occurrence of a creation event for a quantum particle, one can show that the generic component of the spinor associated with the quantum particle under consideration (and thus the quantum potential associated with it) has an important link with the curvature of the ordinary space-time we perceive. This means that the effects of gravity on the geometry and the quantum effects on the geometry of space-time are highly coupled and that both of them can be derived from the RS processes of the 3D timeless non-local quantum vacuum.

From Eq. (28), by changing the ordinary differentiating $\partial_{\mu}$ with the covariant derivative $\nabla_{\mu}$ and by changing the Lorentz metric with the curved metric $g_{\mu \nu}$, one obtains the equations of motion for a change of the quantum vacuum energy density [which determines the occurrence of a creation event for a quantum particle $Q$ of mass (9)] in a curved background:

$\tilde{g}_{\mu \nu} \tilde{\nabla}_{\mu} S_{Q, i} \tilde{\nabla}_{\nu} S_{Q, i}=\frac{V^{2}\left(\Delta \rho_{\mathrm{qvE}}\right)^{2}}{c^{2} \hbar^{2}}$, where $\tilde{\nabla}_{\mu}$ represents the covariant differentiation with respect to the metric

$\tilde{g}_{\mu \nu}=g_{\mu \nu} / \exp Q$

which is a conformal metric, where

$Q_{Q, i}=\frac{\hbar^{2} c^{2}}{V^{2}\left(\Delta \rho_{\mathrm{qvE}}\right)^{2}} \frac{\left(\nabla^{2}-\frac{1}{c^{2}} \frac{\partial^{2}}{\partial t^{2}}\right)_{g}\left|\psi_{Q, i}\right|}{\left|\psi_{Q, i}\right|}$

is the quantum potential of the vacuum.

On the basis of Eqs. (75)-(77), one can say that the quantum potential of the vacuum determined by the RS processes associated with creation events of quantum particles from the $3 \mathrm{D}$ quantum vacuum is equivalent to a curved space-time with its metric given by (76). The quantum potential of the vacuum corresponding to the generic component of the spinor of a quantum particle is tightly linked with the curvature of the space-time we perceive. In other words, we can say that the RS processes, through the manifestation of the quantum potential of the vacuum (77), lead to the generation, at our macroscopic level of reality, of a curvature of space-time. At the same time, the space-time metric is linked with the quantum potential of the vacuum, which influences and determines the behavior of the particles (corresponding to the creation events in the $3 \mathrm{D}$ timeless nonlocal quantum vacuum). In this way, a fundamental geometrization of the quantum aspects of matter is obtained in a picture, where the variable energy density corresponding to the elementary RS processes of the 3D timeless non-local quantum vacuum acting as a superfluid medium is the ultimate entity. In this approach, one can say that the space-time geometry sometimes looks like what we call gravity and sometimes looks like what we understand as quantum behaviors. Both these features of physical geometry emerge from the RS processes of the 3D timeless nonlocal quantum vacuum corresponding to the variable energy density [21].

It is also of interest to observe that the quantum potential of the vacuum (77) equipped with the conformal metric (76) allows us to throw a new light onto the interpretation of the cosmological constant, by taking account for the fruitful considerations made by Ali and Das in [39]. By assuming that the universe is filled by a condensate described by a wave function $\Psi=\mathrm{Re}^{i S / \hbar}$ in the context of the Raychaudhuri equa- 
tion, they found a quantum potential of the form

$Q=\frac{\hbar^{2}}{3 m^{2}} q^{a b} \frac{\left(\nabla^{2}-\frac{1}{c^{2}} \frac{\partial^{2}}{\partial t^{2}}\right) R}{R}$,

where $q_{a b}=g_{a b}-u_{a} u_{b}, u_{a}=\frac{\hbar}{m} \partial_{a} S$. Ali's and Das' quantum potential (78) allows one to obtain directly a cosmological constant that, by assuming a Gaussian form of the wave function or a scalar field theory, is expressed by the relation

$\Lambda_{Q}=\frac{1}{L_{0}^{2}}=(m c / \hbar)^{2}$,

where $L_{0}=\hbar / m c$ may be identified with the current linear dimension of our observable universe, $m$ can be interpreted as the small mass of gravitons (or axions), with gravity (or Coulomb field) ruled by a force law of the Yukawa type

$F=-G \frac{m_{1} m_{2}}{r^{2}} \exp \left(-r / L_{0}\right)$.

In fact, by considering the value $L_{0}=1.4 \times 10^{26} \mathrm{~m}$ and, thus, $m \approx 10^{-68} \mathrm{Kg}$, one obtains the cosmological constant in good agreement with observations:

$\Lambda_{Q}=10^{-52} \mathrm{~m}^{-2}$.

By making a parallelism with Ali's and Das' approach in our approach of Quantum Relativity, the information associated with the quantum potential (77), being linked with the curvature of space-time in virtue of the conformal metric (76), may be assimilated with the quantum potential (78). Therefore, in analogy to Ali's and Das' approach, by assuming a Gaussian form of the wave function of creation events $\Psi \approx \exp \left(-r^{2} / L_{0}^{2}\right)$, where $L_{0}=\frac{\hbar c}{\Delta \rho_{\mathrm{qvE} V}}$, we obtain

$$
\begin{aligned}
& \frac{\hbar^{2} c^{2}}{V^{2}\left(\Delta \rho_{\mathrm{qvE}}\right)^{2}} \frac{\left(\nabla^{2}-\frac{1}{c^{2}} \frac{\partial^{2}}{\partial t^{2}}\right)_{g}\left|\psi_{Q, i}\right|}{\left|\psi_{Q, i}\right|}= \\
& =\frac{1}{L_{0}^{2}}=\left(\frac{\Delta \rho_{\mathrm{qvE}} V}{\hbar c}\right)^{2} .
\end{aligned}
$$

Therefore, taking $L_{0}=1.4 \times 10^{26} \mathrm{~m}$, the corresponding value of the quantum vacuum energy density fluctuations in the minimum quantized space given by the Planck volume is

$\Delta \rho_{\mathrm{qvE}}=\frac{1.4 \times 10^{26} \hbar c}{l_{\mathrm{p}}^{3}}=1.048327 \times 10^{105} \mathrm{~J} / \mathrm{m}^{3}$.
This value is the change of the quantum vacuum energy density, which corresponds to the cosmological constant in good agreement with observations [compatibly with (81)]. The difference of the approach of Quantum Relativity lies in the fact that, here, gravitons (or axions) do not exist as a primary physical reality, but may be seen as a result of the interplay of opportune quantum vacuum energy density fluctuations of the more fundamental variable quantum vacuum energy density.

Furthermore, Quantum Relativity yields the interesting perspective to face the problems of the current Standard Model, by suggesting to consider a general scalar potential in the picture of a 3D timeless quantum vacuum characterized by the elementary processes of creation/annihilation of quanta [40]. In this approach, the most general scalar potential invariant under the Standard Model gauge group has the form

$V=\lambda_{C} C^{4}+\lambda_{I} s_{I}^{4}+\lambda_{R I} s_{I}^{2} s_{R}^{2}+\lambda_{R} s_{R}^{4}+$

$+\lambda_{I C} C^{2} s_{I}^{2}+\lambda_{R C} C^{2} s_{R}^{2}$,

where

$C=\left(\begin{array}{c}\psi_{Q, i} \\ \phi_{Q, i}\end{array}\right)$

is the wave function with two components describing the probability of the occurrence of a creation/destruction event for a quantum particle $Q$ of a given mass (9) (determined by an opportune change $\Delta \rho_{\mathrm{qvE}}$ of the quantum vacuum energy density) in a point event $x, s_{R}$ and $s_{I}$ are the real and imaginary parts of a singlet field $S$, which is a function of the changes and fluctuations of the quantum vacuum energy density, $\lambda_{C}$ is the coupling associated with the wave function $C, \lambda_{R}$ is the coupling associated with the real part $s_{R}$ of the singlet field $S$, and $\lambda_{I}$ is the coupling associated with the imaginary part $s_{I}$ of the singlet field $S$. We have

$\lambda_{R}=\lambda_{S}+\lambda_{S}^{\prime}+\lambda_{S}^{\prime \prime}$

$\lambda_{I}=\lambda_{S}+\lambda_{S}^{\prime}-\lambda_{S}^{\prime \prime}$,

$\lambda_{R I}=2\left(\lambda_{S}-3 \lambda_{S}^{\prime}\right)$,

$\lambda_{R C}=\lambda_{S C}+\lambda_{S C}^{\prime}$,

$\lambda_{I C}=\lambda_{S C}-\lambda_{S C}^{\prime}$

The one-loop renormalization group equations of the scalar couplings in terms of the top Yukawa coupling 
$y_{t}$ and the Standard Model gauge couplings $g$ and $g^{\prime}$ are

$$
\begin{aligned}
& 16 \pi^{2} \beta_{\lambda_{C}}=\frac{3}{8}\left(3 g^{4}+2 g^{2} g^{\prime 2}+g^{\prime 4}\right)+\frac{1}{2}\left(\lambda_{R C}^{2}+\lambda_{I C}^{2}\right)+ \\
& +24 \lambda_{C}^{2}-3 \lambda_{C}\left(3 g^{2}+g^{\prime 2}-4 y_{t}^{2}\right)-6 y_{t}^{4}, \\
& 16 \pi^{2} \beta_{\lambda_{R}}=18 \lambda_{R}^{2}+2 \lambda_{R C}^{2}+\frac{1}{2} \lambda_{R I}^{2}, \\
& 16 \pi^{2} \beta_{\lambda_{I}}=18 \lambda_{I}^{2}+2 \lambda_{I C}^{2}+\frac{1}{2} \lambda_{R I}^{2}, \\
& 16 \pi^{2} \beta_{\lambda_{R I}}=4 \lambda_{I C} \lambda_{R C}+6 \lambda_{R I}\left(\lambda_{I}+\lambda_{R}\right)+4 \lambda_{R I}^{2}, \\
& 16 \pi^{2} \beta_{\lambda_{R C}}=-\frac{3}{2} \lambda_{R C}\left(g^{\prime 2}+3 g^{2}-4 y_{t}^{2}\right)+\lambda_{I C} \lambda_{R I}+ \\
& +6 \lambda_{R C}\left(2 \lambda_{C}+\lambda_{R}\right)+4 \lambda_{R C}^{2}, \\
& 16 \pi^{2} \beta_{\lambda_{I C}}=-\frac{3}{2} \lambda_{I C}\left(g^{\prime 2}+3 g^{2}-4 y_{t}^{2}\right)+\lambda_{R C} \lambda_{R I}+ \\
& +6 \lambda_{I C}\left(2 \lambda_{C}+\lambda_{I}\right)+4 \lambda_{I C}^{2} .
\end{aligned}
$$

By approximating $\lambda_{R}$ as

$\lambda_{R}=\beta_{\lambda_{R}} \ln \frac{\left|s_{R}\right|}{s_{0}}$,

where $\beta_{\lambda_{R}}$ is the always positive beta function of $\lambda_{R}$, and $s_{0}$ is the scale, on which $\lambda_{R}$ becomes negative, the square quantum vacuum energy density matrix for CP-even fields in the basis $\left(C, s_{R}\right)$ is given by

$$
\left(\begin{array}{ll}
2 v^{2} \lambda_{C} & -\sqrt{2} v^{2} \sqrt{\lambda_{C}\left|\lambda_{R C}\right|} \\
-\sqrt{2} v^{2} \sqrt{\lambda_{C}\left|\lambda_{R C}\right|} & \left|\lambda_{R C}\right| v^{2}+\frac{2 \beta_{\lambda_{R}} \lambda_{C} v^{2}}{\left|\lambda_{R C}\right|}
\end{array}\right),
$$

where

$$
v=\frac{s_{0}}{e^{1 / 4}} \sqrt{\frac{\left|\lambda_{R C}\right|}{2 \lambda_{C}}} .
$$

In the case of small $\lambda_{R C}$, the square matrix (97) leads to the following eigenvalues for the energy density of the quantum vacuum:

$\rho_{h}^{2} \cong v^{2}\left(2 \lambda_{C}-\frac{\lambda_{R C}^{2}}{\beta_{\lambda_{R}}}\right)$
$\rho_{s}^{2} \cong v^{2}\left(2 \frac{\beta_{\lambda_{R}} \lambda_{C}}{\left|\lambda_{R C}\right|}+\frac{\lambda_{C}^{2}}{\beta_{\lambda_{R}}}+\left|\lambda_{R C}\right|\right)$

while the CP-odd quantum vacuum energy density is

$\rho_{s}^{2} \cong v^{2}\left(2 \frac{\lambda_{C} \lambda_{R I}}{\left|\lambda_{R C}\right|}+\frac{\lambda_{I C}}{2}\right)$
Equations (99)-(100) are valid only if $\frac{\lambda_{R C}^{2}}{\beta_{\lambda_{R}}} \ll 1$. If this is not true, the eingenvalues of the density matrix (97) for the energy density of the quantum vacuum are:

$\rho_{h}^{2} \cong v^{2}\left(2 \lambda_{C}+\left|\lambda_{R C}\right|+\beta_{\lambda_{R}}\right)$,

$\rho_{s}^{2} \cong v^{2}\left(2 \frac{\beta_{\lambda_{R}} \lambda_{C}}{\left|\lambda_{R C}\right|}+\beta_{\lambda_{R}}\right)$

which imply that the real singlet $s_{R}$ can be derived from a quantum vacuum energy density, which is associated to a mass lighter than the Higgs boson. On the basis of Eqs. (99)-(103), the singlet $s_{R}$ decays to Standard Model particles via more fundamental values of the quantum vacuum energy density. The proposed approach based on Eqs. (99)-(103) implies in this way that the mixing action of the Higgs boson in the production of the mass of Standard Model particles cannot be considered as a fundamental physical reality, but follows from more fundamental entities represented by opportune physical values of the quantum vacuum energy density, given just by Eqs. (99)(103). One can say that, in this picture, at a fundamental level, the Higgs boson does not exist as a physical reality: the action of the Higgs boson is only an emerging reality, it is the interplay of opportune fluctuations of the quantum vacuum energy density, which determines, indeed, the action of the Higgs boson.

Another relevant perspective of this approach lies in the possibility to remove the global minimum of the Standard Model Higgs potential

$V(h)=-\mu^{2} h^{2}+\lambda_{\mathrm{H}}(h) h^{4}$

$\left(-\mu^{2}\right.$ being the Higgs mass parameter, $h$ the Higgs field strength, $\lambda_{\mathrm{H}}$ the Higgs quartic coupling) in the vicinity of the scale $\approx 10^{26} \mathrm{GeV}$ (where $\lambda_{\mathrm{H}}$ runs negative values). In this regard, in analogy to the approach developed by Gabrielli et al. in [41], one can show that the various couplings of the scalar sector have a crucial role in removing the global minimum of the Standard Model Higgs potential (104) and generating the electroweak symmetry breaking minimum.

By following the philosophy that is at the basis of Gabrielli's program, let us start in Quantum Relativity based on the 3D timeless quantum vacuum, by looking at the running of $\lambda_{R}$. We set $\lambda_{R}$ to a small negative value on the electroweak scale. Since the

ISSN 2071-0194. Ukr. J. Phys. 2018. Vol. 63, No. 7

\section{0}


beta-function (91) is always positive, $\lambda_{R}$ will grow, when running toward higher energies and will cross zero on some scale $s_{0}$ above the electroweak scale. This scale is provided by the initial value of $\lambda_{R}$ on the electroweak scale and by the slope of the running set by the beta-function. Since $\lambda_{R}$ itself has to be small near the scale $s_{0}$, and since $\lambda_{R C}$ is required to be small in order to keep the mixing between $s_{R}$ and the regime of small wave function $C$, the betafunction (91) is dominated by $\lambda_{R I}$ on low scales. In order to avoid a huge hierarchy between $s_{0}$ and the electroweak scale, the running of $\lambda_{R}$ has to be sufficiently rapid, implying that $\lambda_{R I}$ cannot be very small.

To remove the global minimum characterizing the Standard Model Higgs potential a positive term has to be added to the beta-function of $\lambda_{C}$. On the basis of Eq. (90), this can be achieved by the term $\lambda_{R C}^{2}+$ $+\lambda_{I C}^{2}$. Since $\lambda_{R C}$ is small to avoid a large mixing, namely this term is dominated by $\lambda_{I C}$, and the global minimum must be removed by setting a sizable initial value for $\lambda_{I C}$ on the electroweak scale.

We want to avoid generating a vacuum expectation value for the imaginary part $s_{I}$, which means that $\lambda_{I}$ must be positive. Hence, we set a small positive initial value for $\lambda_{I}$ on the electroweak scale. Betafunction (92) of $\lambda_{I}$ contains a positive contribution from both $\lambda_{I C}$ and $\lambda_{R I}$, which we know from above to have sizable values. Therefore, the running of $\lambda_{I}$ will be quite rapid, and it will eventually run into a Landau pole. Let us choose the initial values for the parameters on the top mass scale as $\lambda_{R I}=0.3$, $\lambda_{R}=-1.2 \times 10^{-3}, \lambda_{I C}=0.35, \lambda_{I}=0.01, \lambda_{R C}=$ $=-10^{-4}, \lambda_{C}=0.12879$, and $m_{t}=173.1 \mathrm{GeV}$ and let us take the beta functions of the first order in the scalar couplings and the second order in gauge couplings. In this picture, the couplings of this approach yield a Higgs self-coupling $\lambda_{\mathrm{H}}$, which remains positive. Therefore, the Standard Model global minimum at $10^{26} \mathrm{GeV}$ is removed, while $\lambda_{R}$ becomes negative around $s_{0} \approx 10^{4} \mathrm{GeV}$.

On the basis of Eqs. (99)-(103), the electroweak symmetry breaking is indeed determined by the real component of the singlet field $S$ (while the imaginary component remains stable because of the CPinvariance of the general scalar potential (84)). The wave function of the quantum vacuum associated to the occurrence of creation/destruction events, which trigger the electroweak symmetry breaking, can be expressed in the following form:

$C=\exp \left\{i \frac{\sigma_{i}}{2} \theta_{i}(x)\right\} \frac{1}{\sqrt{2}}\left(\begin{array}{l}0 \\ \lambda_{R}+s_{R}(x)\end{array}\right)$.

Now, in analogy to the Standard Model, by defining the covariant derivative as

$D^{\mu} C=\left[\partial^{\mu}+i g \tilde{W}^{\mu}+i g^{\prime} y_{\phi} B^{\mu}\right] C$,

where

$\tilde{W}_{\mu \nu}=\frac{\sigma_{i}}{2} W_{\mu \nu}^{i}, W_{\mu \nu}^{i}=\partial_{\mu} W_{\nu}^{i}-\partial_{\nu} W_{\mu}^{i}-g \varepsilon^{i j k} W_{\mu}^{i} W_{\nu}^{k}$,

$\mathbf{W}_{\mu}$ being the massless isovector triplet (for $S U(2)_{L}$ ), $g$ being the coupling of the isospin current $\mathbf{J}_{\mu}$ of the fermions to $\mathbf{W}_{\mu}, B_{\mu}$ being the mass isosinglet (for $\left.U(1)_{Y}\right), g^{\prime}$ being the coupling of the hypercharge current of the same fermions to $B_{\mu}$, as in the original Weinberg-Glashow-Salam model [42-44]. If one takes the physical unitary gauge $\theta_{i}(x)=0$ with regards for the electroweak symmetry breaking, one obtains

$$
\begin{aligned}
& \left(D^{\mu} C\right)^{+} D^{\mu} C \stackrel{\theta^{i} \rightarrow 0}{\longrightarrow} \frac{1}{2} \partial_{\mu} s_{R} \partial^{\mu} s_{R}+ \\
& +\left(\lambda_{R}+s_{R}\right)^{2}\left\{\frac{g^{2}}{4} W_{\mu}^{+} W^{\mu}+\frac{g^{2}}{8 \cos ^{2} \theta_{W}} Z_{\mu} Z^{\mu}\right\} .
\end{aligned}
$$

The kinetic piece of the Lagrangian in the electroweak symmetry breaking regime (108) leads the vacuum expectation value of the neutral scalar to generate a quadratic term for the gauge bosons $W^{ \pm}$and the $\mathrm{Z}$ and thus leads those gauge bosons to acquire masses on the basis of the relation

$M_{Z} \cos \theta_{W}=M_{W}=\frac{75}{2 \sin \theta_{W}} \mathrm{GeV}=\frac{1}{2} \lambda_{R} g$,

$\theta_{W}$ being the weak mixing angle. Therefore, one finds that, in the electroweak symmetry breaking regime, the coupling $\lambda_{R}$ associated with the real part of the singlet field $S$ has the following expression:

$\lambda_{R}=\frac{75}{g \sin \theta_{W}}$.

The general scalar gauged potential (84) suggests a new interesting manner to give masses to fermions, as well as to the intermediate carriers of the weak force. On the basis of the consideration of the kinetic 
piece (108) in Quantum Relativity, one can define a new effective Lagrangian, which can be defined as a "beyond Standard Model Lagrangian." On the one hand, it is invariant under gauge transformations, which guarantees the renormalizability of the associated quantum field theory (as it occurs in the original Weinberg-Glashow-Salam theory [45]). On the other hand, it allows the electroweak symmetry breaking to occur dynamically via a dimensional transmutation determined by the singlet couplings associated with the singlet field $S$, which is a function of the changes and fluctuations of the energy density of the timeless $3 \mathrm{D}$ quantum vacuum, thus removing the global minimum of the Standard Model Higgs potential. This "beyond Standard Model Lagrangian" is defined as

$$
\begin{aligned}
& L_{\text {effective }}^{\text {beyond } S M}=-\frac{1}{4} W_{\mu \nu}^{a} W^{a \mu \nu}-\frac{1}{4} B_{\mu \nu} B^{\mu \nu}- \\
& -g \bar{C}_{1} \gamma^{\mu} \tilde{W}_{\mu} C_{1}-g \sin \theta_{W} A_{\mu} \sum_{j} \bar{C}_{j} \gamma^{\mu} Q_{j} C_{j}- \\
& -\frac{g}{2 \cos \theta_{W}} J_{\mu}^{Z} Z^{\mu}+\frac{1}{2} \partial_{\mu} s_{R} \partial^{\mu} s_{R}+ \\
& +\left(\lambda_{R}+s_{R}\right)^{2}\left\{\frac{g^{2}}{4} W_{\mu}^{+} W^{\mu}+\frac{g^{2}}{8 \cos ^{2} \theta_{W}} Z_{\mu} Z^{\mu}\right\}- \\
& -t\left(\lambda_{C} C^{4}+\lambda_{I} s_{I}^{4}+\lambda_{R I} s_{I}^{2} s_{R}^{2}+\lambda_{R} s_{R}^{4}+\right. \\
& \left.+\lambda_{I C} C^{2} s_{I}^{2}+\lambda_{R C} C^{2} s_{R}^{2}\right),
\end{aligned}
$$

where $C_{1}$ is the wave function of the quantum vacuum determining the appearance of a left-handed electron and a neutrino in the state $\psi_{1}=\left(\begin{array}{l}\nu_{e} \\ e^{-}\end{array}\right)_{L}$. In the light of the effective Lagrangian, which determines the electroweak Symmetry Breaking dynamically via a dimensional transmutation by means of the couplings associated with the singlet field $S$ of the timeless 3D quantum vacuum, the Vacuum Expectation Value of the scalar sector then induces the Standard Model Higgs Vacuum Expectation Value through a portal coupling [40].

At the end of this section, it is of interest to make some considerations about how our model is presented in a general picture with respect to the Standard Model and what progress it allows us to reach. The Standard Model Lagrangian contains only four parameters, $g, g^{\prime}, \mu^{2}$, and $h$, but it implies the necessity to introduce the Higgs field in an ad hoc form, which yields a global minimum at $\approx 10^{26} \mathrm{GeV}$, thus determining significant problems in this energy range, in the light of Gabrielli's results [41]. Instead, in this model, in the gauge and scalar sectors, the beyond Standard Model Lagrangian allows us to face, in a significant coherent way, different problems encountered by the Standard Model (in connection to the electroweak symmetry breaking and the possibility to remove the global minimum at $\approx 10^{26}$ ) $\mathrm{GeV}$ in the picture of the same $3 \mathrm{D}$ timeless quantum vacuum characterized by fluctuations of its energy density corresponding to the elementary processes of annihilation/creation of quanta. However, these significant results are obtained at the price of the introduction of several physically important parameters, namely the couplings associated with the wave function of the quantum vacuum (but it is worth to mention that, among these, as regards the generation of masses associated with the spontaneous symmetry breaking, the most fundamental parameter is indeed the coupling associated with the real component of the singlet field $S$ ).

\section{Conclusions}

The variable energy density corresponding to the elementary RS processes of creation/annihilation of quanta of the 3D timeless non-local quantum vacuum acting as a superfluid medium is the fundamental background, which determines the Sagnac effect, gravity, dark energy, and quantum behavior of subatomic particles. Interesting perspectives of the unification of gravity and quantum in a sole picture, where a non-local quantum potential of the vacuum is the ultimate entity, and a completion of the Standard Model, where the action of the Higgs boson emerges as the interplay of opportune fluctuations of the quantum vacuum energy density, are opened.

1. C. Rovelli. Loop quantum gravity. Physics World 7 (11), 1 (2003).

2. C. Rovelli. Loop quantum gravity. http:// relativity.livingreviews.org/Articles/lrr-2008-5/ (2008).

3. C. Rovelli. A new look at loop quantum gravity. arXiv: 1004.1780v1 [gr-qc] (2010).

4. D. Fiscaletti, A. Sorli. Perspectives about quantum mechanics in a model of a three-dimensional quantum vacuum where time is a mathematical dimension. SOP Transactions on Theoretical Physics 1 (3), 11 (2014).

5. D. Fiscaletti, A. Sorli. Space-time curvature of general relativity and energy density of a three-dimensional quantum vacuum. Annales UMCS Sectio AAA: Physica LXIX, 55 (2014).

ISSN 2071-0194. Ukr. J. Phys. 2018. Vol. 63, No. 7 
6. D. Fiscaletti, A. Sorli. About a new suggested interpretation of special theory of relativity within a threedimensional Euclid space. Annales UMCS Sectio AAA: Physica LXVIII, 36 (2013).

7. A. Rueda, B. Haisch. Gravity and the quantum vacuum inertia hypothesis. Annalen der Physik 14 (8), 479 (2005).

8. D. Fiscaletti. About dark energy and dark matter in a three-dimensional quantum vacuum model. Foundations of Physics 46 (10), 1307 (2016).

9. D. Fiscaletti, A. Sorli. Bijective epistemology and spacetime. Foundations of Science 20 (4), 387 (2015).

10. D. Fiscaletti. Foreword: The quantum and the geometry. Quantum Matter 3 (3), 161 (2014).

11. I. Licata. Vision of oneness. Spacetime geometry and quantum physics. In Vision of Oneness. Edited by I. Licata, A. Sakaji (Aracne, 2011).

12. L. Chiatti. The transaction as a quantum concept. In Space-Time Geometry and Quantum Events. Edited by I. Licata (Nova Science, 2014).

13. I. Licata. Transaction and non-locality in quantum field theory. European Physical Journal Web of Conferenes 70, 00039 (2014).

14. G.E. Volovik. The Universe in a Helium Droplet (Clarendon Press, 2003).

15. V. Sbitnev. From the Newton's laws to motion of the fluid and superfluid vacuum: vortex tubes, rings, and others. arXiv: 1403.3900v2 [physics.flu-dyn] (2014).

16. V. Sbitnev. Hydrodynamics of the physical vacuum: dark matter is an illusion. arXiv: 1507.03519v1 [physics.gen-ph] (2015).

17. V. Sbitnev. Physical vacuum is a special superfluid medium. In Selected Topics in Applications of Quantum Mechanics. Edited by M.R. Pahlavani (InTech, Rijeka, 2015) Chapter 12, p. 345.

18. V. Sbitnev. Navier-Stokes equation describes the movement of a special superfluid medium. http://arxiv.org/abs/ 1504.07497 (2015).

19. I. Licata, L. Chiatti. Timeless approach to quantum jumps. Quanta 4 (1), 10 (2015).

20. D. Fiscaletti, A. Sorli. About a three-dimensional quantum vacuum as the ultimate origin of gravity, electromagnetic field, dark energy ... and quantum behaviour. $U k r$. J. Phys. 61 (5), 413 (2016).

21. D. Fiscaletti. What is the actual behaviour of the electron? From Bohm's approach to the transactional interpretation to a three-dimensional timeless non-local quantum vacuum. Electron. J. Theor. Phys. 13 (35), 1 (2016).

22. D. Fiscaletti, I. Licata. Bell length in the entanglement geometry. Intern. J. Theor. Phys. 54 (7), 2362 (2015).

23. J.S. Bell. On the Einstein-Podolsky-Rosen paradox. Physics 1 (3), 195 (1964).

24. J.F. Clauser, M.A. Horn, A. Shimony, R.A. Holt. Proposed experiment to test local hidden-variable theories. Phys. Rev. Lett. 23, 880 (1969).
25. J.F. Clauser, M.A. Horne. Experimental consequences of objective local theories. Phys. Rev. D 10, 526 (1974).

26. M.W. Hall. Correlation distance and bounds for mutual information. Entropy 15, 3698 (2013).

27. N. Easwar, D.A. Macintire. Study of the effect of relativistic time dilation on cosmic ray muon flux - An undergraduate modern physics experiment. Am. J. Phys. 59 (7), 589 (1991).

28. E.J. Post. Sagnac effect. Rev. Mod. Phys. 39 (2), 475 (1967).

29. D. Fiscaletti, A. Sorli. Dynamic quantum vacuum and relativity. Annales UMCS Sectio AAA: Physica LXXI, 11 (2016).

30. J.H. Field. The Sagnac effect and transformations of relative velocities between inertial frames. http:// www.relativity-myths.org.uk/jhfield/pdf/sagnacp1rr.pdf (2012).

31. A. Tartaglia. Does anything happen on a rotating disk? In Relativity in Rotating Frames. Edited by G. Rizzi, M.L. Ruggiero (Kluwer, 2004).

32. R.D. Klauber. Derivation of the general case Sagnac result using non-time-orthogonal analysis. Foundat. Phys. Lett. 16 (5), 447 (2003).

33. N. Ashby. Relativity in the global positioning system. Living Reviews in Relativity 6, 1 (2003).

34. A. Rueda, B. Haisch. Contribution to the inertial mass by reaction of the vacuum to accelerated motion. Foundat. Phys. 28 (7), 1057 (1998).

35. A. Rueda, B. Haisch. Inertial mass as reaction of the vacuum to accelerated motion. Phys. Lett. A 240 (3), 115 (1998).

36. B. Haisch, A. Rueda, Y. Dobyns. Inertial mass and the quantum vacuum fields Annalen der Physik (Leipzig) 10 (5), 393 (2001).

37. A. Shojai, F. Shojai. About some problems raised by the relativistic form of de Broglie-Bohm theory of pilot wave. Physica Scripta 64 (5), 413 (2001).

38. F. Shojai, A. Shojai. Understanding quantum theory in terms of geometry. arXiv:gr-qc/0404102 v1 (2004).

39. A.F. Ali, S. Das. Cosmology from quantum potential. Phys. Lett. B 741, 276 (2015)

40. D. Fiscaletti, A. Sorli. About electroweak symmetry breaking, electroweak vacuum and dark matter in a new suggested proposal of completion of the Standard Model in terms of energy fluctuations of a timeless three-dimensional quantum vacuum. Quantum Phys. Lett. 5 (3), 33 (2016).

41. E. Gabrielli, M. Heikinheimo, K. Kannike, A. Racioppi, M. Raidal, C. Spethmann. Towards completing the Standard Model: Vacuum stability, EWSB and dark matter. arXiv: 1309.6632.pdf [hep-th] (2013).

42. S.L. Glashow. Partial-symmetries of weak interactions. $N u$ clear Physics 22 (4), 579 (1961).

43. S. Weinberg. A model of leptons. Phys. Rev. Lett. 19 (21), 1264 (1967)

44. A. Salam. Weak and electromagnetic interactions. Conf. Proc. C 680519, 367 (1968). 
45. G. 't Hooft. Renormalization of massless Yang-Mills fields. Nuclear Physics B 33 (1), 173 (1971).

Д. Фіскалетmi, А. Сорлі

Received 19.08.17

КВАНТОВА ТЕОРІЯ ВІДНОСНОСТІ: ЗМІННА

ЩІЛНІСТЬ ЕНЕРГІЇ КВАНТОВОГО ВАКУУМУ

ЯК ПРИЧИНА МАСИ, ГРАВІТАЦІї

ТА КВАНТОВОЇ ПОВЕДІНКИ

$\mathrm{P}$ е $з$ ю м е

У квантовій теорії відносності (КТВ) час і простір розділені. Час дає числове вираження змін матерії, а простір - це середовище, в якому ці зміни відбуваються. Простір створюється з тривимірного квантового вакууму, який визна- чається флуктуаціями щільності енергії, що відповідає елементарним процесам народження/знищення елементарних квантів у редукованому стані (PC процесам). КТВ дає єдиний підхід до спеціальної теорії відносності, загальної теорії відносності і квантової механіки. Кожен фізичний об'єкт від мікро- до макромасштабу може бути отриманий при відповідному зменшенні щільності енергї квантового вакууму. Зокрема, змінна щільність енергії простору в КТВ відповідає кривизні простору в загальній теорії відносності. В КТВ поведінка кожної субатомной частки випливає з відповідних PC процесів, що залежать від квантового потенціалу вакууму. Проаналізовано перспективи даної моделі для гравітації і квантів як двох сторін однієї монети і електрослабкої взаємодії. 